\title{
3. Die Ära Werner Bock (1949-1963)
}

\subsection{Der harte Kampf um höhere Löhne}

Gewerkschaftliche Kernaufgabe ist die Tarifpolitik. Kollektive Vereinbarungen wirken dem Ungleichgewicht der wirtschaftlichen Macht von einzelnen Arbeitnehmer:innen und dem Arbeitgeber bei der Verteilung des Erarbeiteten entgegen. Die Entgelte der abhängig Beschäftigten haben eine Doppelfunktion: Für die einen sind sie Lebensgrundlage, für die anderen Kosten. Der Ertrag des gemeinsam Erarbeiteten fließt zunächst in die Kassen der Unternehmen und die Gewerkschaften haben die Aufgabe, den Beschäftigten den ihnen zustehenden Anteil daraus zu sichern.

Dieser Aushandlungsprozess gelingt in den meisten Fällen ohne größeren Streit. Verweigern Arbeitgeber den Beschäftigten jedoch den nach Ansicht der Gewerkschaften "gerechten Anteil«, können die Arbeitnehmer:innen ihre Arbeitsleistung vorübergehend verweigern, haben also den Streik als Druckmittel. Heute wird die Bereitschaft zu streiken in der Regel durch befristete Warnstreiks demonstriert. Kommt es weiterhin zu keiner Einigung, lassen die Gewerkschaften ihre Mitglieder über einen dann normalerweise unbefristeten Streik abstimmen, was als Urabstimmung bezeichnet wird. Bei einem unbefristeten Streik geht es für beide Seiten »ums Eingemachte«, deshalb kommen bei einem Arbeitskampf stets auch Emotionen, ja sogar Leidenschaft mit ins Spiel.

Den rechtlichen Rahmen für die autonome kollektive Gestaltung von Arbeitsund Wirtschaftsbedingungen setzt die Verfassung mit nur wenigen Worten. Weitere Regulierungen im deutschen Arbeitskampfrecht haben nach und nach Gerichte geschaffen.

In der Textil- und der Bekleidungsindustrie wurde die Tarifpolitik durch die frühen wirtschaftlichen Krisen der Branchen erschwert, zugleich waren die Erwartungen der Gewerkschaftsmitglieder angesichts eines relativ niedrigen Branchenlohnniveaus hoch - seit Beginn der Industrialisierung lagen die Verdienste in der Textil- und der Bekleidungsindustrie im unteren Bereich der Lohnskala.

Die GTB verfolgte in den ersten beiden Nachkriegsjahrzehnten konsequent die Linie, den Lohnabstand zwischen den von ihr betreuten Branchen und dem Durchschnitt der Industrielöhne zu verringern. Die Arbeitgeber wurden mit dem 
von DGB-Chefvolkswirt Viktor Agartz entwickelten Konzept der »expansiven Lohnpolitik« konfrontiert, das auf den Erfahrungen der US-amerikanischen Gewerkschaften fußte (siehe Kapitel 3.1.6). Die Gegensätze bei der Beurteilung des tarifpolitisch Machbaren, aber auch die tief verankerte Gegnerschaft von Unternehmern und Gewerkschaft, mündeten in harten Arbeitskämpfen. Dabei setzte sich die GTB trotz der oft nicht rosigen Wirtschaftslage weitgehend durch, da sie geschlossener handelte als das Arbeitgeberlager. Erst zum Ende der 1950er Jahre setzte ein Nachdenken über breitere, innovative Ansätze der Tarifpolitik ein.

\subsubsection{Theorie und Praxis des Koalitionsrechts}

Unter »Koalitionsrecht« ist die Freiheit von Beschäftigten wie Arbeitgebern zu verstehen, sich zusammenzuschließen, um Arbeitsbedingungen für abhängig Beschäftigte kollektiv zu regeln. Historisch gesehen wurde dieses Recht von der Arbeitnehmerseite erkämpft. So ist der Lohnstreik der Webergesellen in Speyer im Jahr 1351 einer der ersten bekannten Arbeitskämpfe in Deutschland. ${ }^{1}$

Seit dem Mittelalter verboten oder eingeschränkt, wurde die Koalitionsfreiheit im Deutschen Reich schließlich ab 1872 gewährt und sicherte damit die rechtliche Grundlage für die Bildung von Gewerkschaften, die sich schon vorher, meist aus Arbeiterbildungsvereinen, gegründet hatten. Dennoch gab es anfangs viele Behinderungen und die zwischen 1878 und 1889 geltenden Sozialistengesetze bremsten die Entwicklung. Nach der Revolution von 1918 wurde das Koalitionsrecht 1919 in die Weimarer Verfassung aufgenommen und 1933 durch das nationalsozialistische Regime aufgehoben. 1949 fand es als Grundrecht Eingang in das Grundgesetz der Bundesrepublik Deutschland, ${ }^{2}$ wo es in Artikel 9 Absatz 3 heißt:

»Das Recht, zur Wahrung und Förderung der Arbeits- und Wirtschaftsbedingungen Vereinigungen zu bilden, ist für jedermann und für alle Berufe gewährleistet. Abreden, die dieses Recht einschränken oder zu behindern suchen, sind nichtig, hierauf gerichtete Maßnahmen sind rechtswidrig. ॥ $^{3}$

Dieses Recht beinhaltet sowohl die individuelle Freiheit »für jedermann«, eine Koalition zu bilden bzw. ihr beizutreten, als auch den Bestands- und Betätigungsschutz der Koalitionen und damit auch das Streikrecht. Der Staat garantiert einen

1 Kittner 2005: Arbeitskampf, S. 25.

2 In die DDR-Verfassung wurde das Koalitionsrecht ebenfalls aufgenommen und durch das Streikrecht der Gewerkschaften ergänzt. Angesichts eines der SED vollständig unterstellten EinheitsGewerkschaftsbundes blieb es jedoch reine Makulatur. Das Streikrecht wurde dann mit der Verfassungsänderung 1968 stillschweigend gestrichen.

3 Zit. nach https://www.gesetze-im-internet.de/gg/BJNRoooo10949.html (Abruf am 25.1.2021). 
Freiraum, in dem Akteur:innen die Bedingungen abhängiger Arbeit autonom verhandeln und vereinbaren können - die Kurzformel hierfür lautet Tarifautonomie. Das Grundrecht auf Koalitionsfreiheit verpflichtet nicht nur die Träger öffentlicher Gewalt, sondern ist auch eine Schutzbestimmung gegenüber Dritten.

Die GTB definierte das Recht zur Regelung der »Arbeits- und Wirtschaftsbedingungen« so, dass es weit mehr als die direkten materiellen Arbeitsbedingungen wie Einkommen und Arbeitszeit umfasst. Als Gewerkschaft hatte die GTB den Anspruch, Themen wie die Gestaltung von Rationalisierungsfolgen, die Arbeitsplatzgestaltung, die Anwendung von Leistungslohnsystemen, aber auch die Zusammenarbeit mit Arbeitgeberverbänden über die Tarifrunden hinaus vertraglich mit den Arbeitgebern zu regeln. ${ }^{4}$

Das aus Artikel 9 Absatz 3 Grundgesetz abgeleitete Streikrecht ist in Deutschland nicht weiter gesetzlich geregelt; den sich daraus ergebenden Freiraum mussten die Gerichte gestalten. Anfang der 1950er Jahre war die rechtliche Rahmensetzung noch schwach ausgestaltet und uneinheitlich. Das Bundesarbeitsgericht wurde erst durch das im September 1953 verabschiedete Arbeitsgerichtsgesetz geschaffen. Das Tarifvertragsgesetz, das noch vor Gründung der Bundesrepublik durch den Wirtschaftsrat des Vereinigten Wirtschaftsgebietes der britischen und amerikanischen Besatzungszone beschlossen wurde, regelte nur die formalen Bedingungen für das Tarifgeschehen.

\subsubsection{Die Tarifpolitik der Anfangsjahre}

Im Fokus der gewerkschaftlichen Aufgaben der ersten Jahre standen die Einkommen. Die Kontrollratsdirektive Nr. 14 der Besatzungsmächte hatte für alle Löhne oberhalb von 50 Pfennig pro Stunde einen Lohnstopp verhängt. Dieser galt auch für die Textil- und die Bekleidungsindustrie. Doch die drei westlichen Besatzungsmächte hatten die Direktive unterschiedlich angewendet und beispielsweise Lohnerhöhungen für Frauen und Jugendliche zugelassen. Der erste Hauptvorstand der GTB musste 1949 zunächst Leitlinien für eine Tarifpolitik erarbeiten, konkrete Forderungen entwickeln und die sehr regionalen Strukturen zumindest koordinieren. ${ }^{5}$

Die Textilindustrie war zwar der erste Wirtschaftszweig gewesen, der industrialisiert wurde, was aber nicht bedeutete, dass die Löhne in dieser Branche hoch waren. Im Gegenteil bestand und besteht bis heute eine deutliche Verdienstlücke im Vergleich zum industriellen Durchschnitt. Der Geschäftsbericht der GTB vermerkt für den Juni 1950 eine Lohnlücke in der Textilindustrie von 8,8 Prozent zum

\footnotetext{
4 So in den Rationalisierungsschutzabkommen von 1964 für die Textilindustrie und von 1988 für die Textil- und die Bekleidungsindustrie wie auch im Manteltarif Bekleidungsindustrie von 1979.

5 CTB: Geschäftsbericht 1949-1950 des Hauptvorstandes, S. 55 f.
} 
Industriedurchschnitt. ${ }^{6}$ Für den November 1952 wird bei den männlichen Arbeitern in der Textilindustrie eine Differenz von 17 Prozent zum industriellen Durchschnitt genannt, während die Frauen 4 Prozent über dem Industriedurchschnitt lagen. ${ }^{7}$ Das Statistische Bundesamt weist die ältesten Vergleichszahlen erst für das Jahr 1957 aus. Demnach lagen in diesem Jahr die Löhne in der Textilindustrie um 22,1 Prozent und die der Bekleidungsindustrie um 31,8 Prozent unter denen des gesamten Verarbeitenden Gewerbes.

Die ausschlaggebende Ursache hierfür war der hohe Frauenanteil der Branchen, der in der Textilindustrie in den 1950er Jahren 65 Prozent, in der Bekleidungsindustrie 85 Prozent betrug. Der Anteil der Frauen an den in der Gesamtwirtschaft Beschäftigten lag dagegen bei nur 31 Prozent. ${ }^{8}$ Die Arbeitszeit betrug in der frühen Nachkriegszeit 48 Stunden wöchentlich und die Arbeitsbedingungen waren belastend. In der Textilindustrie war Schichtarbeit die Regel; neben der körperlich anstrengenden Arbeit setzten Feuchtigkeit, Staub, Lärm und Vibrationen den Beschäftigten zu. In der Bekleidungsindustrie war Bandarbeit üblich. In der arbeitsteiligen Fertigung hieß das, dass man bzw. frau im Arbeitsfluss mithalten musste. Die physische und psychische Belastung traf besonders die Frauen, da auf die meisten von ihnen nach der Erwerbsarbeit noch die Familienarbeit zu Hause wartete.

Zuständig für die Tarifpolitik in der Textilindustrie waren innerhalb der GTB die damaligen neun Bezirksleitungen. In der ersten Konstitutions- und Konsolidierungsphase der Nachkriegszeit waren die Tarifverhandlungen noch stark lokal geprägt, mit kleinen und kleinsten Tarifgebieten, die in ihrer Vielfalt kaum zentral zu koordinieren waren. Erst 1952 gelang es der GTB, in allen Tarifgebieten Tarifverträge durchzusetzen. Immer wieder kam es zu lokalen Streiks. Nachdem die Strukturen der GTB bundesweit stabiler waren, wurden die Kämpfe organisierter und in größeren Tarifgebieten geführt.

Bis Mitte der 1950er Jahre wurden die Tarifverträge durchweg auf der Grundlage der Arbeitsordnungen aus nationalsozialistischer Zeit vereinbart, die wiederum ihre Basis in den Tarifverträgen der 1920er Jahre hatten. Neue tarifliche Strukturen wurden ab Mitte der 1950er Jahre erst Schritt für Schritt geschaffen, die teilweise lokalen Vereinbarungen der Textilindustrie auf Länder- oder TeilLänderebene ${ }^{9}$ vereinheitlicht. Für die Bekleidungsindustrie wurden von Beginn an ein Bundes-Lohntarifvertrag und bundesweit geltende Manteltarifvertrags-

6 GTB: Geschäftsbericht 1949-1950 des Hauptvorstandes, S. 61.

7 GTB: Geschäftsbericht 1951-1952 des Hauptvorstandes, S. 71.

8 Statistisches Bundesamt (1952): Wirtschaft und Statistik, 4. Jahrgang, Heft 8, S. 304.

9 So gab es unterschiedliche Tarifverträge für Nord- und Südbayern, für Nordrhein und für Westfalen. 
bestimmungen vereinbart. Die regionalen Arbeitgeberverbände der Bekleidungsindustrie übertrugen dazu ihre Verhandlungsmandate an den BBI. ${ }^{10}$

Die branchenwirtschaftlichen Bedingungen waren bei der Verbesserung der Lohn- und Arbeitsbedingungen wenig hilfreich. Die Textilindustrie entwickelte sich spürbar anders als die Gesamtwirtschaft, die Koreakrise 1951/1952 und eine Überproduktionskrise 1957/1958 führten zu scharfen Umsatz- und Produktionseinbrüchen. Die Unternehmen, die 1950 Lohnzuwächse von über 10 Prozent zugestanden hatten, versuchten bereits im Zuge der ersten Krise, Lohnkürzungen durchzusetzen. So stockte die Tarifentwicklung schon Mitte 1951. Da die meisten Beschäftigten im Akkord arbeiteten, kamen vorzugsweise Kürzungen der Leistungslohnvorgaben zum Zuge.

Hierbei war entscheidend, dass die Arbeitgeber die Regelungen des 1952 in Kraft getretenen Betriebsverfassungsgesetzes nicht akzeptierten. Nach $\$ 56$ dieses Gesetzes hatte der Betriebsrat, soweit eine tarifliche Regelung nicht bestand, »bei der Regelung von Akkord- und Stücklohnsätzen « mitzubestimmen. ${ }^{11}$ Die Arbeitgeber argumentierten in zwei Richtungen: Einerseits verwiesen sie auf die noch geltenden Tarifordnungen (anstelle von Manteltarifverträgen), durch die es eine tarifliche Regelung gebe, die diese Mitbestimmung nicht vorsehe. Andererseits behaupteten sie, Stücklöhne seien einzelarbeitsvertragliche Regelungen: Setze der Arbeitgeber einen Stücklohn fest und beginne der Arbeitnehmer bzw. die Arbeitnehmerin zu arbeiten, sei durch Angebot und Annahme ein Einzelarbeitsvertrag mit ebendieser Kondition zustande gekommen. In beiden Fällen verneinten sie die Mitbestimmung von Betriebsräten. ${ }^{12}$

Diese Verbiegung des Rechts konterkarierte die Tarifpolitik und führte immer wieder zu betrieblichen Konflikten, die teilweise in Arbeitskämpfen mündeten. Dennoch dauerte der Streit um das Mitbestimmungsrecht der Betriebsräte viele Jahre, bis das Bundesarbeitsgericht 1955 im Sinne der GTB entschied. ${ }^{13}$

Auch im Zeitlohn Beschäftigte blieben von Lohnkürzungen nicht verschont. Die Belegschaften waren wegen der »Gutsherren-Mentalität« der Unternehmer oft in Aufruhr. Aufgrund von Akkordkürzungen kam es bereits 1950-1952 zu einer Reihe spontaner betrieblicher Arbeitsniederlegungen. Die Streiks gingen oftmals von Betriebsräten oder gewerkschaftlichen Vertrauensleuten in den Betrieben aus und wurden dann erst von der GTB »übernommen«, d.h. die Arbeitskampfmaßnahmen wurden im Nachhinein von der Gewerkschaft genehmigt. Karl Buschmann, damals im GTB-Vorstand für Tarifpolitik zuständig, warf den

10 Zum Bundesverband Bekleidungsindustrie (BBI) siehe Kapitel 2.3.2.

11 Veröffentlicht in: Bundesgesetzblatt, Teil I, 11.10.1952, S. 688.

12 Laut Vortrag des Verbandssyndikus Keller vor dem Hauptausschuss von Gesamttextil am 14. November 1952, BWA No2 61.

13 BAG, 12.10.1955-1 ABR 13/54. 
Unternehmern auf dem Gewerkschaftstag 1953 vor, die betrieblichen Löhne zum Ausgleichsfonds bei wirtschaftlichen Krisen zu machen und den kapitalistischen Gewinnabsichten unterzuordnen. ${ }^{14}$

Die in ihren Grundsätzen aus der Zeit der Weimarer Republik stammenden Tarifstrukturen waren im Vergleich mit heutigen Tarifverträgen sehr einfach aufgebaut und unterschieden hauptsächlich zwischen gelernten und ungelernten Arbeiter:innen. Die Mehrzahl der Beschäftigten, nämlich die Frauen, wurden nicht nur indirekt diskriminiert, vielmehr sahen die Tarifverträge für sie auch niedrigere Löhne für die gleiche Tätigkeit vor. Die Arbeitgeber vertraten nachdrücklich die Auffassung, dass der Gleichheitsgrundsatz des Grundgesetzes nicht auf Tarifverträge anzuwenden war. ${ }^{15}$

Trotz vieler aufgezwungener Abwehrkämpfe nahm sich die GTB in der Tarifpolitik folgende Schwerpunkte vor: ${ }^{16}$

- Einkommenserhöhung und Krisensicherheit der Löhne bei Verringerung des Abstandes zum industriellen Durchschnitt

- Angleichung der Frauenlöhne

- Arbeitszeitverkürzung mit dem Ziel der 40-Stunden-Woche

- Umgestaltung der auf den Tarifordnungen basierten Tarifverträge mit dem Ziel, die Tätigkeiten zu bewerten, statt die formale Qualifikation als Grundlage zu nehmen

\subsubsection{Der Streik von 1953}

Anfang 1953 stockten die Tarifverhandlungen in fast allen Tarifgebieten. Der GTB-Forderung nach einer Erhöhung der Stundenlöhne um 12 Pfennig für alle Arbeiter:innen stellten die Arbeitgeber das Angebot von 7 Pfennig ausschließlich für die im Zeitlohn Beschäftigten entgegen. Gesamttextil hatte die Arbeitgeber eingeschworen, bei Akkord- und Stücklohnsätzen keinerlei Zugeständnisse zu machen. ${ }^{17}$ Der »Herr-im-Hause-Standpunkt « sollte unbedingt verteidigt werden, was für die 80 Prozent der Arbeiter:innen, die im Akkordlohn standen, bedeutete, dass sie leer ausgehen würden. Daraufhin traten am 26. Januar 1953 drei Betriebe im südbadischen Zell im Wiesental in den Streik. Die Bemühungen der GTB, diesen Streik auszuweiten, blieben erfolglos.

\footnotetext{
14 GTB: Protokoll des 3. Ordentlichen Cewerkschaftstages, 15.-19. Juni 1953 in Düsseldorf, S. 142.

15 CTB: Ceschäftsbericht 1951-1952 des Hauptvorstandes, S. $173 \mathrm{f}$.

16 Vgl. Buschmann (1953): Lohn- und Tarifpolitik in Gegenwart und Zukunft; CTB: Protokoll des 4. Ordentlichen Cewerkschaftstages, 5.-8. Juli 1955 in Frankfurt am Main, S. 366 f. (EntschlieBung zur Lohn- und Tarifpolitik).

17 Cesamttextil: Vertrauliches Rundschreiben vom 20. November 1952, BWA No2 60.
} 
Eine größere Dimension erreichte der Konflikt im Tarifgebiet Westfalen/Regierungsbezirk Osnabrück. Insbesondere das westliche Westfalen, wo der Streit in der Hauptsache ausgetragen wurde, war ländlich-katholisch geprägt. Der freigewerkschaftliche Textilarbeiter-Verband hatte hier in der Weimarer Zeit kaum eine Rolle gespielt. Viele führende GTB-Gewerkschafter, auch der Bezirksleiter, waren in den christlichen Vorläuferorganisationen sozialisiert und aktuell der CDU verbunden. Dennoch hatten die Textilbetriebe eine starke gewerkschaftliche Tradition mit hohem Organisationsgrad. Vor diesem Hintergrund war klar, dass hier kein vorder- oder hintergründig ideologisch begründeter Arbeitskampf geführt werden würde. Vielmehr ging es darum, auch als Textiler:in am Wirtschaftsaufschwung teilzuhaben, nachdem es 1952 vor dem Hintergrund der Koreakrise nicht gelungen war, Tarifverbesserungen durchzusetzen.

Der Streik begann am 28. Januar 1953 und entwickelte sich zum härtesten Arbeitskampf der jungen Bundesrepublik. 47 Prozent aller bundesdeutschen Streikausfalltage des Jahres entfielen auf ihn, ${ }^{18}$ über 23.000 Streikende beteiligten sich. In Nordhorn an der niederländischen Grenze, das durch drei große Textilunternehmen geprägt war, traten die Arbeiter:innen als Erstes in den Streik.

Während die niedersächsische Stadt angesichts des Streiks »den Atem anhielt«, wie die »Grafschafter Nachrichten« schrieben, ging es in den Kleinstädten des Münsterlandes konfliktreicher zu. Hier blockierten Streikposten nicht nur Streikbrecher:innen, sondern auch Auslieferungen von Fertigware und Rohstoffanlieferungen. Die Arbeitgeber forderten immer wieder Polizei an. Diese und die politisch Verantwortlichen zeigten sich angesichts dieser für die ländliche Region absolut ungewohnten Situation völlig überfordert. So ging die Polizei mit gezückten Pistolen, Gummiknüppeleinsätzen und berittenen Ordnungskräften gegen Streikende und Streikposten vor, wobei immer wieder Personen verletzt wurden. ${ }^{19}$ Die GTB-Mitgliederzeitung berichtete:

»So rasten aber Einsatzwagen mit Ta-tü-ta-ta durch die sonst so stillen Städtchen und Orte des Münsterlandes. Man witterte Aufruhr, Aufstand und Umsturz. Arbeitgeber sahen sich bedroht und begaben sich und ihr >Eigentum< unter polizeilichen Schutz. Wenn einige Bürger zusammenstanden, so waren das erklärte Ansammlungen. Mehrere Bürger-wohlgemerkt nicht Streikende-bezahlten ihre >Neugierdeくmit Nasenstübern und Kostproben des Cummiknüppels. ${ }^{20}$

Arbeitgeber drohten mit der Kündigung von Werkswohnungen, Arbeitnehmerdarlehen und dem Abbau »freiwilliger« Sozialleistungen. Es hagelte Anzeigen

18 Spode et al. (1992): Statistik der Arbeitskämpfe in Deutschland.

19 textil-bekleidung, Ausgabe Ende Februar 1953, S. 1.

20 textil-bekleidung, Ausgabe Mitte März 1953, S. 1. 
gegen Mitglieder von Streikleitungen. Unorganisierte wurden aufgefordert, Schadensersatzklagen gegen die GTB zu erheben, deren Mitglieder ihnen den Weg zur Arbeit versperrt hätten. Die lokale Presse unkte von kommunistischen Unterwanderungen, denen die Arbeiter:innen auf dem Leim gegangen seien.

Mehr als sechs Wochen lang bewegten sich die Unternehmer keinen Millimeter. Schließlich gelang es dem nordrhein-westfälischen Arbeitsminister Johann Ernst, die Tarifparteien wieder an den Tisch zu bekommen und einen Kompromiss durchzusetzen. Dieser sah eine Erhöhung von Stundenlöhnen und Akkordrichtsätzen um 7 bis 9 Pfennig rückwirkend ab 1. Januar 1953 vor. Als Besonderheit gab es die höchste Lohnerhöhung für die unterste Lohngruppe der Hilfsarbeiter:innen, deren Lohn sich um 8,7 Prozent erhöhte, während die Facharbeiterlöhne nur um 5,9 Prozent stiegen. Der westfälische Arbeitgeberverband schrieb dem Minister daraufhin, die Auftragslage sei nach wie vor äußerst ungünstig. Aufgrund des Preisdrucks durch starke Liberalisierung der Importe sei eine allgemeine Lohnsteigerung in keiner Weise gerechtfertigt:

»Die Gewerkschaft hat in völliger Verkennung der Lage einen Streik begonnen, der in seiner Durchführung Zeichen organisierten Terrors gezeitigt und dadurch die an sich schon schweren Schäden für die betroffenen Arbeiter, für die Betriebe und für die ganze Volkswirtschaft noch verstärkt hat.«

Unter erheblichen Bedenken stimme der Vorstand dem Vorschlag dennoch zu. ${ }^{21}$ Auf der anderen Seite empfanden viele Streikende das Ergebnis angesichts des entbehrungsreichen Kampfes und der ursprünglichen Forderung von 12 Pfennig als Niederlage. Eine Delegiertenversammlung, in der die Vertretungen aus nicht am Streik beteiligten Betrieben die Mehrheit hatten, nahm das Verhandlungsergebnis am 11. März 1953 an und am 12. März wurde die Arbeit wieder aufgenommen.

Eine Reihe von aktiv am Streik Beteiligten wurde nicht wieder eingestellt. Die Arbeitgeber legten die Maßregelungsklausel, nach der bestimmt war, dass niemand wegen der Beteiligung am Streik entlassen oder gemaßregelt werden dürfe, eng aus. Wer aktiver gewesen war, als sich nur beteiligt zu haben, also Streikposten stand oder in der Streikleitung mitgewirkt hatte, sei durch die Klausel nicht geschützt. ${ }^{22}$ So waren es insbesondere Betriebsratsmitglieder und gewerkschaftliche Vertrauensleute, die den Streik durch Arbeitsplatzverlust zu bezahlen hatten. Ein Jahr später bat der GTB-Vorsitzende Werner Bock den Verbandspräsiden-

21 Schreiben des Verbandes der Textilindustrie Westfalen vom 11. März 1953 an den Arbeitsminister des Landes Nordrhein-Westfalen, BWA No2 61.

22 Unterstützt wurde diese Auffassung durch ein Rechtsgutachten von Prof. Rolf Dietz, BWA No2 62. 
ten Willy van Delden darum, sich um eine Wiedereinstellung der immer noch 41 arbeitslosen Gewerkschafter zu bemühen. ${ }^{23}$

Auch straf- und zivilrechtlich wurde gegen Beschäftigte vorgegangen. Es gab rund 100 Anklagen wegen Landfriedensbruchs, Nötigung, Aufruhr, Beleidigung oder Körperverletzung. Überwiegend erfolgten Freisprüche und Einstellungen der Verfahren, in einem Fall wurde eine dreimonatige Freiheitsstrafe verhängt, in rund einem Dutzend der Fälle wurden die Gewerkschafter zu Geldstrafen zwischen 20 und 300 DM verurteilt. Die Arbeitgeber hatten Arbeitswillige aufgefordert, wegen ihres Lohnausfalls Klagen auf Schadensersatz zu stellen. Dem kamen einige nach und traten ihre Forderungen dann an ihre Arbeitgeber ab, welche sie wiederum an den Arbeitgeberverband abtraten. Dieser vertrat nun vor Gericht die Arbeiter, die den Streik brechen wollten. Auch Firmen außerhalb des Streikgebiets klagten auf Schadenersatz, weil für sie vorgesehene Lieferungen blockiert worden waren. ${ }^{24}$

Dieser Streik, so umstritten das Ergebnis auch bei den Gewerkschaftsmitgliedern aufgenommen wurde, war für die weiteren Beziehungen der Tarifparteien von anhaltender Bedeutung. Die Arbeitgeber waren von der Durchsetzungsfähigkeit der GTB tief beeindruckt. Dies galt umso mehr, als das Münsterland geradezu mustergültig für die traditionelle Textilindustrie mit ihren alteingesessenen patriarchalischen Strukturen stand. Die Firmeninhaber sahen sich als gütige, wenn auch strenge Oberhäupter der Firmengemeinschaft und verkrafteten es nur schwer, von ihren Arbeiter:innen derart enttäuscht zu werden. Gesamttextil verschärfte als Reaktion auf den Streik die tarifpolitische Koordination.

Bei dem parallel laufenden Streik im südbadischen Zell hatten die Arbeitgeber erklärt, jegliche Verhandlungen abzulehnen, solange noch gestreikt wird. Damit hatten sie Erfolg: Am 7. Februar 1953 stimmten 62 Prozent der Gewerkschaftsmitglieder dafür, zunächst die Arbeit wieder aufzunehmen. Auf Arbeitgeberseite sollte diese Strategie künftig bei allen Streiks durchgehalten werden: In allen Tarifgebieten sollten Verhandlungen verweigert werden, sofern noch in irgendeinem Tarifgebiet gestreikt würde. Außerdem wurde der für Tarifpolitik zuständige Sozialpolitische Ausschuss von Gesamttextil gestärkt und seine schnelle Reaktionsfähigkeit durch ein größeres Maß an Selbstständigkeit erhöht. Die regionalen Arbeitgeberverbände richteten mit der Gründung einer "Gefahrengemeinschaft « zudem ein Unterstützungssystem für bestreikte Unternehmen ein. ${ }^{25}$

Auch in der GTB setzte sich die Erkenntnis durch, dass eine bessere Koordination nötig war. Während des Streiks in Westfalen hatte die Bezirksleitung Nord-

23 AdsD 5/GTBA100149.

24

25

GTB: Geschäftsbericht 1953-1954 des Hauptvorstandes, S. $177 \mathrm{f}$.

Gesamttextil: Protokoll der Sitzung des Sozialpolitischen Ausschusses vom 5./6. Juni 1953, BWA No2 61. 
bayern am 13. Februar 1953 einen Tarifvertrag mit Lohnerhöhungen zwischen 5 und 6 Pfennig abgeschlossen, die zudem erst ab 1. März 1953 wirksam werden sollten, obwohl der Lohntarifvertrag zum 31. Dezember 1952 gekündigt worden war. Auch für Rheinland-Pfalz war am 30. Januar 1953 ein Tarifabschluss unterschrieben worden, der dann aber auf Druck des Hauptvorstandes widerrufen wurde. Solch unsolidarisches Verhalten müsse für die Zukunft ausgeschlossen sein, forderte Buschmann im GTB-Beirat. ${ }^{26}$ Nach dem westfälischen Abschluss wurde die allgemeine Tariferhöhung von 7 Pfennig in allen Tarifgebieten umgesetzt, die außerordentliche Verbesserung für die Hilfsarbeiter:innen gab es jedoch nur im Streikbezirk selbst.

Bedeutend war der Arbeitskampf in Westfalen/Osnabrück auch aus politischen Gründen. Das relativ neue Modell der Einheitsgewerkschaft GTB hatte sich bewährt - trotz versuchter Einflussnahme von KPD und SED auf der einen und Konservativen auf der anderen Seite. Vor allem die »christlichen« Kolleg:innen hatten bewiesen, dass sie keine Schwachstelle in der Durchsetzungsfähigkeit der Einheitsgewerkschaft waren, sondern sechs Wochen lang einen der härtesten Arbeitskämpfe der Nachkriegsjahre geführt hatten.

\subsubsection{Reform der Tarifverträge und Angleichung der Frauenlöhne}

Neben dem Bestreben nach Beteiligung der Arbeiter:innen an der wachsenden Produktivität trieb die GTB in der ersten Hälfte der 1950er Jahre die Reform der Tarifverträge voran. Im Fokus standen tätigkeitsbezogene Eingruppierungen, die Erhöhung der Anzahl der Lohngruppen - und dadurch eine bessere Durchlässigkeit für Höhergruppierungen - sowie die Abschaffung der Frauenlöhne. Der Respekt, den sich die GTB mit dem Westfalenstreik erarbeitet hatte, aber auch die ab 1954 einsetzende Textilkonjunktur verbesserten die Voraussetzungen zur Durchsetzung dieser Vorhaben.

Bereits bevor das Bundesarbeitsgericht (BAG) im Januar 1955 die Rechtswidrigkeit der bis dahin üblichen Frauenlohngruppen mit niedrigerer Vergütung verkündete, gelang in der Bekleidungsindustrie Anfang Dezember 1954 die Abschaffung dieser diskriminierenden Tarifvertragsbestimmungen. Nicht mehr das Geschlecht, sondern die ausgeübte Tätigkeit bestimmte von nun an die Eingruppierung. In der Textilindustrie versuchten die Arbeitgeber, die im Tarifvertrag verankerte zwanzigprozentige Lohndifferenz zu rechtfertigen und mit allen Mitteln zu verteidigen. Sie bestritten, dass Frauen bei gleicher Arbeit die gleiche Leistung erbringen würden: Die schwächere physische und psychische Konstitution, aber auch Dauer der Betriebszugehörigkeit und Umfang der Berufsausbil-

26 GTB: Protokoll der Beiratssitzung vom 16./17. Februar 1953, AdsD 5/CTBA0301001. 
dung, Fehlzeiten sowie Sonderbedingungen des Arbeits- und Arbeitsschutzrechtes wurden als Rechtfertigung für die Ungleichbehandlung ins Feld geführt. ${ }^{27}$

Vom Urteil des Bundesarbeitsgerichts, das die pauschale Schlechterbezahlung von Frauen als verfassungswidrig und damit für nichtig erklärte, ${ }^{28}$ zeigten sich die Arbeitgeber überrascht. Ernsthaft wurde diskutiert, Druck für eine Verfassungsänderung zu machen, was jedoch mehrheitlich als wenig erfolgversprechend angesehen wurde. Verständigen konnte man sich im Sozialpolitischen Ausschuss von Gesamttextil aber auf die Argumentation zur Verteidigung von Lohndifferenzen,

»dass die Mehrzahl der weiblichen Arbeitnehmer ihren Arbeitsauftrag recht und schlecht erfülle, während die Mehrzahl der Männer entsprechend ihrer größeren Leistungsfähigkeit, Zuverlässigkeit, Selbständigkeit und vielseitigerer Verwendbarkeit einen Anspruch auf einen höheren Tariflohn geltend machen könne «. ${ }^{29}$

Bei der Umsetzung des BAG-Urteils auch in den Tarifgebieten der Textilindustrie gelang es der GTB, sogenannte Leichtlohngruppen zu verhindern, die die Arbeitgeber als Ersatz für die Frauenlohngruppen durchsetzen wollten. Sie schlugen vor, künftig geschlechtsunabhängig nur noch zwischen leichten und schweren bzw. einfachen und schwierigen Tätigkeiten zu unterscheiden. Für leichte und einfache Tätigkeiten sollten die bisherigen Frauenlöhne gelten, wodurch sich für die Arbeitgeber die Möglichkeit ergeben hätte, einen Teil der Männer in bisherige Frauenlohngruppen herabzustufen.

Da die Tariflohndifferenz erheblich war, bedeutete die Anhebung der Frauenlöhne für die Textil- und die Bekleidungsindustrie mit ihrem hohen Frauenanteil eine deutliche Lohnkostenerhöhung. Beispielsweise betrug der Stundenlohn für weibliche Hilfskräfte im Tarifgebiet Westfalen 101 Pfennig, für männliche 120 Pfennig; die Sätze bei Facharbeit lagen bei 114 bzw. 134 Pfennig. Im Tarifgebiet Niedersachsen/Bremen der Textilindustrie wurde die Lohngruppe für ungelernte Frauen (87 Pfennig pro Stunde) abgeschafft. Die nunmehr niedrigste Lohngruppe für einfache Tätigkeiten lag bei 112 Pfennig, was einer Erhöhung von 29 Prozent entsprach. Durchschnittlich erhöhten sich die Tariflöhne für Frauen um 20 Prozent. ${ }^{30}$ Der Versuch der GTB, nicht nur diese Differenz auszugleichen, sondern

27 BDA, Protokoll der Sitzung des Arbeitskreises Frauenlohn vom 27. März 1953, BWA No2 61.

28 BAC, 15.1.1955, 1 AZR 305/54.

29 Cesamttextil: Protokoll der Sitzung des Sozialpolitischen Ausschusses vom 10. Februar 1955, BWA No2 65.

30 GTB: Geschäftsbericht 1955-1956 des Hauptvorstandes, S. 102. 
auch gleichwertige Tätigkeiten gleich einzustufen, gelang allerdings nur ansatzweise. ${ }^{31}$

Für Frauen stiegen die Tariflöhne also deutlich. Dennoch blieben viele Hindernisse auf dem Weg zur gleichen Bezahlung. Selbst in den reformierten Tarifverträgen fand sich noch die besondere Betonung schwerer Arbeiten und die festgeschriebene Höherwertigkeit von klassischen Männertätigkeiten. So war im Tarifvertrag für die westfälische Textilindustrie die Tätigkeit »Nähen« mit in der Regel mindestens zweijähriger Ausbildung in der Lohngruppe za eingestuft, während die Tätigkeit »Weben« bei gleicher Ausbildungszeit mit mindestens Lohngruppe 4a vergütet wurde. Die zur Angleichung der tatsächlichen Frauenlöhne unternommenen Schritte blieben also klein, so dass das Thema kontinuierlich auf der Tagesordnung stand.

Zwischen 1954 und 1960 verringerte sich der Lohnabstand der durchschnittlichen Frauenlöhne in der Textilindustrie im Vergleich zu den Männerlöhnen nur von 23,8 Prozent auf 20,8 Prozent, in der Bekleidungsindustrie immerhin von 33,2 Prozent auf 27,0 Prozent. ${ }^{32}$ Trotz der strukturellen Tarifverbesserungen ist die geringe Steigerung ein Indiz dafür, dass Leistungslöhne beschnitten wurden oder zu niedrige Eingruppierungen der Frauen erfolgten. Aber bei den Verantwortlichen der GTB blieb sicher nicht außer Acht, dass die Produktivitätsfortschritte nur einmal verteilt werden können und eine stärkere Verbesserung der Frauenlöhne zulasten der Erhöhungen bei den Männern gegangen wäre.

Die Strukturreformen bei den Tarifverträgen Mitte der 1950er Jahre betrafen nicht nur die Gleichstellung. Sie hoben auch die alte, einfache Trennung »ungelernt, angelernt, gelernt« auf und stellten auf die Tätigkeiten ab. Eine größere Anzahl an Lohngruppen erhöhte auch die Durchlässigkeit; beispielsweise sah der westfälische Tarifvertrag nun 21 Lohnstufen vor. Die in dieser Zeit geschaffenen Strukturen galten unverändert bis in die 1970er Jahre und prägen die Lohnrahmenbestimmungen bis heute.

1955 gelang ein weiterer Erfolg, der die GTB-Tarifpolitik entscheidend stabilisierte. Nach einem zweiwöchigen Streik in der hessischen Textilindustrie konnte erstmals die Effektivlohnklausel durchgesetzt werden. Sie verpflichtete die Arbeitgeber, die vereinbarten Lohnerhöhungen auch tatsächlich den Beschäftigten zugutekommen zu lassen. Eine Anrechnung übertariflicher Lohnbestandteile auf die Tariflohnerhöhung war ab jetzt nur noch durch Änderungskündigung möglich - eine Gewerkschaftsforderung, die Gesamttextil zuvor eher nachlässig zur Kenntnis genommen hatte.

31 CTB: Geschäftsbericht 1955-1956 des Hauptvorstandes, S. 100.

32 Eigene Berechnungen auf Crundlage der Ceschäftsberichte des GTB-Hauptvorstandes. 
Im Dezember 1955 wurde das Arbeitgeberlager davon überrascht, dass dieses Anliegen zur zentralen Forderung wurde und einen Streik auslöste. ${ }^{33}$ Zuvor hatte man eine solche Klausel intern für rechtlich nicht möglich gehalten und stand jetzt vor dem Dilemma, den selbst unterzeichneten Tarifvertrag anzugreifen oder aber mit der neuen Situation zu leben. Immerhin wurde im Sozialpolitischen Ausschuss von Gesamttextil kritisch angemerkt, dass man es bei der Nicht-Weitergabe von Tariferhöhungen mittels Anrechnung übertariflicher Bestandteile wahrscheinlich übertrieben habe. ${ }^{34}$

Die Tarifpolitik der GTB wurde außerdem durch die Lösung der jahrelangen Streitfrage des Mitbestimmungsrechts bei der Festsetzung von Prämien- und Akkordvorgaben sowie Stücklöhnen gestärkt. Am 1. Februar 1957 bestärkte das Bundesarbeitsgericht seine bereits im Oktober 1955 grundsätzlich verkündete Rechtsauffassung und erklärte einseitige Maßnahmen des Arbeitgebers bei der Leistungslohngestaltung, also die Veränderung von Leistungslöhnen, für unwirksam. ${ }^{35}$ Damit entschied das höchste deutsche Arbeitsgericht zugunsten eines Mitbestimmungsrecht des Betriebsrats bei der Festsetzung von Akkord- und Stücklohnsätzen, das die Arbeitgeber und ihre Verbände bisher bestritten hatten.

Im November/Dezember 1956 gelang die Vereinbarung der 45-Stunden-Woche ab 1957 nach dem Muster des »Bremer Abkommens« für die Metallindustrie vom vorangegangenen Juni. Die Arbeitszeitverkürzung war insbesondere ein Anliegen der Frauen, da das traditionelle Rollenbild, also Hausarbeit gleich Frauenarbeit, zu dieser Zeit gesellschaftlich kaum infrage gestellt wurde. Die Frauen waren durch die vollschichtige Erwerbsarbeit an sechs Tagen pro Woche und ihre sogenannte familiäre Reproduktionsarbeit enorm belastet. Daher war die Arbeitszeitverkürzung eine zentrale Frage, auf die die wenigen Frauen in den Entscheidungsgremien nachdrücklich drängten. ${ }^{36}$ Neben der Verkürzung der Wochenarbeitszeit wurde in den textilen Branchen ein Schwerpunkt auf die Verlängerung des Urlaubs gelegt.

Schon 1957 ging die Aufschwungphase in der Textilindustrie, die 1953 eingesetzt hatte, wieder zu Ende. Dunkle Wolken hatten sich am textilen Himmel aufgetürmt. Technologische Verbesserungen der Spinn- und Webmaschinen hatten die Produktivität, aber auch die Produktion deutlich erhöht. Zudem verstärkte

33 Gesamttextil: Protokoll der Sitzung des Sozialpolitischen Ausschusses vom 13. Dezember 1955, BWA No2 68.

34 Gesamttextil: Protokoll der Sitzung des Sozialpolitischen Ausschusses vom 13. Dezember 1955, BWA No2 68.

35 BAG, 01.02.1957-1 AZR 521/54.

36 So Paula Müller, die Mitglied des CTB-Beirats war und auf die baldige Einführung der 40-Stunden-Woche drängte, notfalls auch ohne vollen Lohnausgleich; GTB: Protokoll der Beiratssitzung vom 24./25. Mai 1956, AdsD 5/CTBA0301001. 
sich der außenwirtschaftliche Druck, denn die deutschen Textilimporte verdoppelten sich von 1956 bis $1959 .{ }^{37}$ Obwohl die Unternehmen weitere technische Rationalisierungsmaßnahmen vornahmen, fand der erhöhte Ausstoß der deutschen Textilfabriken keinen Absatz mehr. Die Textilunternehmen reagierten klassisch, Entlassungen und Kurzarbeit prägten die textilen Zentren. Angesichts dieser Lage versuchten die Arbeitgeber, die Lohnentwicklung wieder zu bremsen, aber die GTB hielt an ihrem Ziel fest, den Lohnabstand zum Durchschnitt des Verarbeitenden Gewerbes zu verringern. In einer zurückhaltenden Lohnpolitik sah sie kein Instrument, die Beschäftigung zu stabilisieren.

\subsubsection{Der Streik von 1958}

Anfang 1958 wollten die Arbeitgeber bei den Verhandlungen für das Tarifgebiet Niedersachsen/Bremen die Kürzung der Akkordzuschläge und den Wegfall der Effektivlohnklausel erreichen. Die GTB forderte 25 Pfennig für alle Lohngruppen, was einer Erhöhung von fast 20 Prozent entsprach. Die Textilgewerkschaft hatte sich fest vorgenommen, in dieser Tarifrunde einen Schritt zur Angleichung an das gesamtindustrielle Lohnniveau zu schaffen. Deshalb wurde diese Tarifrunde insbesondere in Niedersachsen und Hessen geradezu generalstabsmäßig vorbereitet. ${ }^{38}$

Im Vergleich zur Metallindustrie waren die Hürden für einen Arbeitskampf bei der GTB deutlich niedriger. Urabstimmungen über einen Streik wurden nicht im gesamten Tarifbezirk, sondern nur in den für den Streik bestimmten Betrieben durchgeführt. Damit war in den gezielt ausgewählten Betrieben eine hohe Zustimmung so gut wie sicher. Die Arbeitgeber der Textilindustrie hatten sich zum Ziel gesetzt, die für die Metallindustrie vereinbarte fünfprozentige Erhöhung nicht zu überschreiten. ${ }^{39}$

Nach Scheitern einer Schlichtungsverhandlung am 28. Januar 1958, bei der die Arbeitgeber eine Erhöhung von 6-9 Pfennig pro Stunde anboten, wurde in vier Bremer und Delmenhorster Betrieben über einen Streik abgestimmt. Unmittelbar nach der Stimmauszählung, bei der eine Zustimmung zwischen 85 und 95 Prozent erreicht wurde, legten die Textilarbeiter:innen die Arbeit nieder. In wenigen Tagen wurde der Streik dann auf Betriebe in ganz Niedersachsen ausgedehnt, insgesamt 9.000 Beschäftigte legten die Arbeit nieder. Ihre Streikparole lautete: "Wir wollen nicht länger Stiefkinder des Wirtschaftswunders sein."

Zwei Wochen später traten auch 6.000 Beschäftigte in Hessen in den Streik. Hier ging es gleichfalls um eine Stundenlohnerhöhung um bis zu 25 Pfennig.

37 Eigene Berechnungen auf Grundlage der Geschäftsberichte des GTB-Hauptvorstandes.

38 CTB (1991): textil-bekleidung. 100 Jahre GTB. Sonderausgabe zum Jubiläum, S. 142.

39 CTB: Protokoll der Beiratssitzung vom 3./4. April 1958, AdsD 5/CTBA0301002. 
Beide Tarifkämpfe wurden, wie bereits der Arbeitskampf 1953 in Westfalen, mit großer Härte geführt. Personalchefs machten Hausbesuche und versuchten, die Ehepartner:innen zu beeinflussen. Sie drohten mit der Kündigung von Werkswohnungen. Auch wenn die Löcher in den Portemonnaies immer größer wurden, kämpften die Textiler:innen geschlossen weiter. Die Streikleitung zählte während des insgesamt neunwöchigen Streiks nur 123 Streikbrecher:innen. ${ }^{40}$

Die regionalen Besonderheiten sowie die Konkurrenz unter den Bezirksleitungen der GTB führten trotz der anderslautenden Verabredungen, die nach dem Streik von 1953 getroffen worden waren (siehe Kapitel 3.1.3), zu Unstimmigkeiten. So gab es in der 1958er Tarifrunde bereits Tarifabschlüsse in anderen Bezirken, während in Niedersachsen/Bremen und Hessen noch gestreikt wurde. Diese lagen zwar oberhalb der Arbeitgeber-Tabu-Grenze von 5 Prozent, jedoch deutlich unterhalb der in den Streikbezirken geforderten 25 Pfennig. So wurde während der siebten Streikwoche in Niedersachsen und Bremen für das große Tarifgebiet Westfalen/Osnabrück eine Erhöhung von 12-15 Pfennig vereinbart, obwohl GTBintern ein Limit von 17 Pfennig verabredet war.

Mit welch harten Bandagen gekämpft wurde, verdeutlicht die Tatsache, dass die niedersächsischen Arbeitgeber die 15-Pfennig-Erhöhung sofort an die nicht Streikenden weitergaben, um die regionale Streikfront zu schwächen. Die GTB konterte, indem sie die Streikgelder um 15,5 Pfennig pro Stunde erhöhte. Am 19. März schließlich ließen sich die Arbeitgeber auf eine Erhöhung um 17 Pfennig für alle Lohngruppen ein, was einem Volumen zwischen 11,5 Prozent und 17 Prozent entsprach. Nach einer Urabstimmung, bei der 77,9 Prozent das Ergebnis annahmen, wurde die Arbeit in den Textilbetrieben wieder aufgenommen. Die Arbeitgeber in Hessen schlossen sich dem norddeutschen Ergebnis zunächst nicht an. Es brauchte eine weitere Streikwoche, bis hier eine Lohnerhöhung von 14-17 Pfennig durchgesetzt wurde. ${ }^{41}$

Die GTB erlebte während der Arbeitskämpfe große Unterstützung vonseiten der DGB-Gewerkschaften und der SPD. Die Arbeitgeber erhielten Unterstützung aus der Politik. Bundeswirtschaftsminister Ludwig Erhard kritisierte in einem Zeitungsbeitrag explizit den GTB-Streik und die Gewerkschaftsforderungen, deren »Durchsetzung zwangsläufig zu einer Schwächung, ja Gefährdung der deutschen Wettbewerbskraft führen müssten ${ }^{42}$. Der Wirtschaftsminister kritisierte gleichzeitig die Unternehmer wegen ihrer zu weichen Position. Diese waren

40 textil-bekleidung, Ausgabe 1/1988, S. 6f. (»Streik-Ceorg Drescher erinnert sich«).

41 GTB: Geschäftsbericht 1957-1958 des Hauptvorstandes, S. 295.

42 Erhard (1958): Es liegt jetzt an uns, ob wir bestehen werden, in: Die Zeit, 20.2.1958. 
Stammgäste im Ministerium und hatten schon 1957 vehement mehr Schutz vor Textilimporten gefordert. ${ }^{43}$

Erhard warf ihnen nun vor, mehr Schutz vor internationaler Konkurrenz zu fordern, um gewerkschaftlichen Forderungen entgegenkommen zu können, und führte aus: »Ich schwöre, daß ich mich zu einer derart verbrecherischen Politik nicht verstehen werde.« Dezidiert kritisierte er die Textilstreiks, die ein Vernichtungswettbewerb seien, und forderte ein Ende der »atomisierten Lohnpolitik, wie sie in der Bundesrepublik in der Selbstverantwortlichkeit der einzelnen Industriegewerkschaften geübt wird«, um zu einer koordinierten Tarifpolitik zu kommen, in der die Tarifpolitik »nicht an den produktivsten Wirtschaftszweigen ausgerichtet sein darf «. ${ }^{44}$

Die GTB hielt an ihrem Ziel der Lohnangleichung zum Industriedurchschnitt fest. Stolz weist der Geschäftsbericht auf die Erhöhungen der Textillöhne hin, denn im Zeitraum 1958-1960 stiegen die Stundenlöhne in der Textilindustrie bei Männern um 26 Prozent und bei Frauen um 32 Prozent. Das waren Steigerungsraten, wie sie seit 1950 nicht erreicht worden waren und die den Industriedurchschnitt mit Erhöhungen um 23 Prozent bei Männern und 29 Prozent bei Frauen übertrafen..$^{45}$ Darüber hinaus war es gelungen, den Urlaub um drei Tage auf nunmehr mindestens drei Wochen zu verlängern und die Wochenarbeitszeit um eine Stunde auf 43 Stunden zu verkürzen.

In den 1950er Jahren war die GTB bezogen auf ihre Größe die streikfreudigste Gewerkschaft der jungen Bundesrepublik. In absoluten Zahlen lag sie bei den Streiktagen nach der IG Metall an zweiter Stelle. ${ }^{46}$ Die Arbeitskämpfe 1953 und 1958 waren im Hinblick auf Beteiligung, Länge und Kosten die größten in der 49-jährigen Geschichte der GTB. 1953 wies die amtliche Streikstatistik im Textilbereich 741.000 streikbedingte Ausfalltage aus; 1958 waren es "nur« 555.000 Tage, was damals jedoch 68 Prozent aller bundesdeutschen Streikausfalltage bedeutete. ${ }^{47}$ Für den Streik 1953 musste die GTB 4 Millionen DM aufbringen, 1958 lagen

43 Im Jahr 1957 gab es einen intensiven Schriftwechsel des Cesamttextil-Präsidenten Carl Neumann mit Ludwig Erhard. Neumann warf dem Bundeswirtschaftsminister in rüdem Ton Zollsenkungen vor, durch die Textilimporte verbilligt würden; Erhard konterte, den Verbandsforderungen jeweils weit entgegengekommen zu sein, jedoch mache er seine Entscheidungen nicht von der Zustimmung durch Cesamttextil abhängig; BWA No2 56.

44 Erhard (1958): Es liegt jetzt an uns, ob wir bestehen werden, in: Die Zeit, 20.2.1958.

45 Statistisches Bundesamt (Destatis) (2020): Verdiensterhebung im Produzierenden Gewerbe 1950-1995.

46 Gertschen (2013): Klassenfeinde-Branchenpartner?, S. 95.

47 Spode et al. (1992): Statistik der Arbeitskämpfe in Deutschland. 
die Kosten bei 6,7 Millionen $\mathrm{DM}^{48}$ und machten damit die Hälfte der Beitragseinnahmen des gesamten Jahres aus. ${ }^{49}$

Durch ihre Taktik der selektiven Urabstimmungen und Streiks ausschließlich in Schwerpunktbetrieben hatte die GTB in den Auseinandersetzungen eine starke Position, da die Individualinteressen der Unternehmer einen geschlossenen Widerstand auf Arbeitgeberseite verhinderten. Im eigenen Unternehmen mochte man nicht auf Zusatzumsätze wegen eines bestreikten Konkurrenzbetriebes verzichten. 1959 klagte Hermann Zeiler, Vorsitzender des Arbeitgeberkreises von Gesamttextil:

"Aufs neue hat sich erwiesen, daß das deutsche Unternehmertum noch immer nicht bereit ist, wirklich solidarisch zu handeln und die einzige Waffe zu führen, die es dem Streik gegenüber gibt: die Aussperrung [...]. Wir konnten diese [...] Waffe nicht führen, einfach, weil sie nicht besteht. Aber damit fehlt uns auch jene Waffengleichheit, fehlt die Parität der Kräfte, die stillschweigend vorausgesetzt war, als unsere Verfassung den Lohn und die sonstigen Arbeitsbedingungen dem freien Aushandeln zwischen autonomen Tarifpartnern übertrug. $\ll^{50}$

Mit ihrer kampfbetonten Tarifpolitik konnte die GTB den Lohnabstand zu anderen Industriezweigen tatsächlich verkleinern. Die Differenz der Stundenlöhne in der Textilindustrie zum Industriedurchschnitt verringerte sich von 20 Prozent Mitte der 1950er Jahre auf 15 Prozent im Jahr 1960. Die Stundenlöhne der Frauen stiegen bis 1960 auf 105 Prozent des industriellen Durchschnitts. ${ }^{51}$

\subsubsection{Das Konzept der "expansiven Lohnpolitik" und innovative Tarifpolitik jenseits des Lohnkampfes}

Trotz der tarifpolitischen Erfolge der GTB in den 1950er Jahren verstärkte sich gegen Ende des Jahrzehnts die interne Diskussion über den Kurs gegenüber den Arbeitgebern. Die kämpferische Politik der GTB wirkte sich negativ auf die Mitgliederzahlen aus, der gewerkschaftliche Organisationsgrad sank, die Beitragseinnahmen stagnierten. Bis dahin hatte sich die Lohnpolitik der GTB an den Thesen von Viktor Agartz ausgerichtet.

48 Aufstellung der Abteilung Finanzen der CTB vom 22.8.1978; AdsD 5/CTBA410265

49 Laut Geschäftsbericht betrug die Jahresbeitragseinnahme 1958 13,9 Millionen DM; vgl. GTB: Geschäftsbericht 1957-1958 des Hauptvorstandes.

50 Cesamttextil: Sitzung des Hauptausschuss vom 8. Dezember 1959, BWA No2 92.

51 Statistisches Bundesamt (Destatis) (2020): Verdiensterhebung im Produzierenden Gewerbe 1950-1995. 
Von 1948 bis 1955 Leiter des Wirtschaftswissenschaftlichen Instituts des Deutschen Gewerkschaftsbundes (WWI), war Agartz maßgeblich an der Entwicklung der gewerkschaftlichen Programmatik beteiligt. Nach seinem Konzept der »expansiven Lohnpolitik" hatten Tarifverhandlungen nicht nur sozialpolitische Wirkung, vielmehr sei jede expandierende Wirtschaft von der Gefahr bedroht, dass die Nachfrage hinter dem Warenangebot zurückbleibe. Daher sollte sich die Lohnpolitik laut Agartz nicht nur an der Produktivität orientieren, sondern versuchen, »die wirtschaftliche Expansion von sich aus zu forcieren, um durch eine bewußte Kaufkraftsteigerung eine Ausweitung der Produktion herauszufordern «. ${ }^{52}$ Zugleich sah Agartz in der expansiven Lohnpolitik keine egoistische Interessenpolitik, sondern eine wachstumsfördernde strukturpolitische Erweiterung der damals keynesianisch geprägten Vorstellungen des ökonomischen Mainstreams.

Trotz aller Systemkritik setzte Agartz darauf, die Produktivkräfte des Kapitalismus zu fördern, um sie für die Beschäftigten zu nutzen. In seinem Hauptreferat auf dem GTB-Gewerkschaftstag 1955 beschrieb er die wirtschaftliche Entwicklung der Textilindustrie als »außerordentlich bescheiden«. Er forderte größere Betriebseinheiten und diese grundsätzlich $\mathrm{zu}$ rationalisieren, um die Kosten durch Spezialisierung und Serienfertigung auf ein Mindestmaß zu senken. Die hohen Löhne der US-amerikanischen Automobilarbeiter und die preiswerten Autos seien schließlich zwei Seiten derselben Medaille; die Struktur der alten Textilländer in Europa sei demgegenüber rückständig. Internationaler Konkurrenz werde mit administrativen Mitteln begegnet, anstatt die Möglichkeiten des marktwirtschaftlichen Wettbewerbs zu nutzen. Agartz prognostizierte, dass sich bald ausrechnen lasse, »wann die Textilindustrie der europäischen Länder eines Tages zu erliegen kommt «.53

Trotz der beschriebenen bescheidenen wirtschaftlichen Lage der Textilindustrie war Agartz über die dort herrschende Einkommenssituation geradezu erschüttert und stellte die rhetorische Frage:

"Soll nun die Lohnquote der in solchen Betrieben beschäftigten Arbeitnehmer die weichere Stelle sein, über die man eine Sanierung durchführt, ohne daß die Beschäftigten in irgendeiner Weise für diese Zustände verantwortlich gemacht werden können? ${ }^{54}$

Die Lohnfrage sah Agartz in unmittelbarem Zusammenhang mit einer Neuordnung der Branche. Die keineswegs beneidenswerte Aufgabe der GTB-Führung sei, umfassend zu handeln:

52 Zit. nach: Der Spiegel (1954): Mindestens das Doppelte, in: Ausgabe 33 vom 11.8.1954, S. 5.

53 CTB: Protokoll des 4. Ordentlichen Kongresses, 5.-8. Juli 1955 in Frankfurt am Main, S. 184.

54 GTB: Protokoll des 4. Ordentlichen Kongresses, 5.-8. Juli 1955 in Frankfurt am Main, S. 188. 
»Höhere Löhne und Strukturänderung der Textilindustrie, kürzere Arbeitszeit und Produktionsplanung, Spezialisierung und Serienfertigung. Das alles ist eine Einheit und eine einheitliche Aufgabe ihrer Gewerkschaft. $\aleph^{55}$

Das Protokoll vermerkte am Ende des Referats »Bravo!«-Rufe und »lebhaften anhaltenden Beifall «. ${ }^{56}$ Agartz hatte den Delegierten, die mit den Schwierigkeiten der Branche täglich konfrontiert waren, aus dem Herzen gesprochen, auch wenn unklar blieb, wie diese »umfassende« Aufgabe angesichts der realen Machtverhältnisse in praktisches Handeln umgesetzt werden konnte. Zum Zeitpunkt dieses Referats beim GTB-Kongress war Agartz, der auf dem DGB-Kongress 1954 noch umjubelte Cheftheoretiker der Gewerkschaften, wegen seines radikalen Konzeptes zur gesellschaftlichen Umgestaltung bereits heftig umstritten.

Karl Buschmann, seit 1951 für die Tarifpolitik der GTB verantwortlich, vertrat unabhängig vom ideologischen Hintergrund die gleiche Richtung der Lohnpolitik wie Agartz. Anfang 1954 bezog er sich in einer Beiratssitzung auf dessen Konzept der »expansiven Lohnpolitik« und das Gremium beschloss, dieses zur Leitlinie der GTB zu machen. ${ }^{57}$

Buschmanns Handeln war unverkennbar von den Erfahrungen der US-amerikanischen Gewerkschaften beeinflusst. Die dortigen Gewerkschaftsverbände AFL und CIO pflegten intensive Kontakte zur bundesdeutschen Arbeiterbewegung und unterhielten eine regelrechte Botschaft, um über ihre Experten Erfahrungen zu vermitteln. Ein grundsätzliches Ziel war, und hier stimmten sie mit der US-Außenpolitik überein, kommunistische Einflüsse zurückzudrängen und die bundesdeutschen Gewerkschaften vom Weg einer konsenskapitalistischen Wirtschaftsordnung zu überzeugen. ${ }^{58}$

So waren auch alle führenden Gewerkschafter der GTB zu Studienreisen in den USA gewesen. Unter diesem Eindruck verfasste Karl Buschmann umfangreiche Berichte, in denen er den hohen gewerkschaftlichen Organisationsgrad, die oftmals kompromisslos eingesetzte Durchsetzungsmacht, aber auch die Akzep$\operatorname{tanz}$ von Rationalisierungen herausstellte. Gewerkschaften in den USA, so stellte er fest, befürworteten technische Neuerungen, wenn diese drei Bedingungen erfüllten: höhere Löhne, höhere Gewinne und niedrigere Produktionskosten, also preisgünstigere Produkte. ${ }^{59}$

Trotz seiner Funktion als ranghöchster GTB-Tarifpolitiker blieb Buschmann in den Arbeitskämpfen der 1950er Jahre eher im Hintergrund. Er beschäftigte

55 GTB: Protokoll des 4. Ordentlichen Kongresses, 5.-8. Juli 1955 in Frankfurt am Main, S. 188.

56 GTB: Protokoll des 4. Ordentlichen Kongresses, 5.-8. Juli 1955 in Frankfurt am Main, S. 189.

57 GTB: Protokoll der Beiratssitzung vom 26./27. Februar 1954, AdsD 5/GTBA0301001.

58 Angster (2003): Konsenskapitalismus und Sozialdemokratie, S. $112 \mathrm{f}$.

59 CTB: Protokoll der Beiratssitzung vom 24./25. Juni 1952, AdsD 5/GTBA0301001. 
sich stark mit der wirtschaftlichen Entwicklung der Textil- und der Bekleidungsindustrie, über die er umfangreich auf den Gewerkschaftstagen berichtete. $\mathrm{Ab}$ Mitte der 1950er Jahre versuchte er, neben der Lohnpolitik auch neue tarifpolitische Akzente zu setzen. So forderte Buschmann auf dem Gewerkschaftstag 1957 die Einrichtung von tariflichen Sozialfonds, weil die neuen Maschinen in der Textilindustrie viele Arbeitsplätze überflüssig machten. Daher sollten die betroffenen Beschäftigten aus einem von den Arbeitgebern gespeisten Fonds unterstützt werden, Fondsverwaltung und Auszahlungen sollte die GTB übernehmen. Dieser Fonds war vor allem zur Unterstützung älterer Beschäftigter gedacht, da es die Jüngeren in Zeiten des allgemeinen Wirtschaftsaufschwungs leichter hatten, in anderen Unternehmen oder Branchen Arbeit zu finden.

Buschmann betonte auf dem Gewerkschaftstag 1957 »die positive Einstellung der Gewerkschaften zur technischen Entwicklung«, für die er jedoch die Bedingung stellte, »die Voraussetzungen dafür [zu] schaffen, daß dem arbeitenden Menschen ein Höchstmaß an sozialer Sicherheit und Gerechtigkeit gewährleistet wird $\lll{ }^{60}$ Ende der 1950er Jahre strebte er ein weitgehendes Rationalisierungsschutzabkommen an, in dem der Grundsatz »Versetzung geht vor Entlassung“ festgeschrieben werden sollte. Sollte das im Betrieb nicht möglich sein, müssten rechtzeitig Freistellungen für Umschulungen sowie Abfindungen garantiert werden, über 50-Jährige sollten generell vor Kündigungen geschützt werden.

Mit diesen über die Lohn- und Arbeitszeitpolitik hinausgehenden tarifpolitischen Ansätzen verband Buschmann mehr als nur die Schutzwirkung für die Beschäftigten. Sein Anliegen war, die Position der GTB als Akteurin in der Branche auszubauen. Die Übernahme sozialpolitischer Aufgaben sollte ein Schritt zur Festigung und Entwicklung der eigenen Organisation werden. Buschmanns Kalkül war, dass sich die GTB über eine Verbreiterung der tarifpolitischen Handlungsfelder innerhalb des Systems der sozialen Marktwirtschaft eine bedeutende Rolle sichern könnte. Er hatte registriert, dass der gewerkschaftliche Organisationsgrad trotz aller tarifpolitischen Erfolge gesunken war. Deshalb setzte sich der Tarifpolitiker mit seinen neuen Ansätzen vorsichtig von der bisherigen, ausschließlich verteilungspolitisch ausgerichteten Tarifpolitik ab. Dass seine tarifpolitischen Visionen auch zu gemeinsamen Einrichtungen mit den Arbeitgeberverbänden führen könnten, war für die GTB ein neuer und eher sozialpartnerschaftlich geprägter Ansatz.

Bisher gelang es nur in bescheidenem Maße, die wachsende Bekleidungsindustrie gewerkschaftlich zu erschließen. Auch in den prosperierenden Textilbetrieben funktionierte es oft nicht, Neueingestellte von einer GTB-Mitgliedschaft zu überzeugen. Daher begann auf dem GTB-Kongress 1957 eine Diskussion,

60 Buschmann (1957): Die technische Entwicklung in der Textilwirtschaft und ihre Auswirkungen auf die Arbeitnehmerschaft, S. 103. 
die acht Jahre später in einen Großkonflikt münden sollte. Tarifverträge, die juristisch ohnehin nur für Gewerkschaftsmitglieder galten, sollten in bestimmtem Maße tatsächlich exklusiv Gewerkschaftsmitgliedern zugutekommen. Damit sollte der Nachteil der Mitglieder, die ja erst durch ihre Beitragsleistung Tarifverträge ermöglichten, ausgeglichen werden. Denn bis heute profitieren Nichtmitglieder vielfach als »Trittbrettfahrer« von den gewerkschaftlich erkämpften tariflichen Leistungen.

\section{2 "Modernisierer" gegen "Traditionalisten «}

Die GTB-Führung der 1950er Jahre war durch ihre Erfahrungen in der Weimarer Republik und der anschließenden Zeit des Nationalsozialismus geprägt, also der Niederlage der Arbeiterbewegung und Zerschlagung der Gewerkschaften. Die in der freien Gewerkschaftsbewegung sozialisierten Mitglieder des Geschäftsführenden Hauptvorstandes (GHV) sahen Unternehmer auch in der Nachkriegszeit tendenziell als Ausbeuter, die es, zumindest temporär, zu besiegen galt - eine Ansicht, die sich auf Arbeitgeberseite widerspiegelte.

Auch Hugo Karpf und Bernhard Tacke, die beiden Christlich-Sozialen im GHV, standen in der unmittelbaren Nachkriegszeit für eine wirtschaftliche Neuausrichtung mit einem hohen Anteil von Gemeineigentum und einer starken betrieblichen und überbetrieblichen Mitbestimmung. Das 1947 verabschiedete "Ahlener Programm« der CDU lehnte die kapitalistische Wirtschaftsordnung als gescheitert $\mathrm{ab}$ und fordert eine gemeinwirtschaftlich orientierte Ordnung. ${ }^{61} 1949$ wurden die Weichen jedoch nach dem Sieg Adenauers über seinen SPD-Konkurrenten Kurt Schumacher in eine andere Richtung gestellt. Diese Aussicht auf die Realisierung von Investitionslenkung und weitreichender Mitbestimmung war bei realistischer Betrachtung spätestens mit der Bundestagswahl von 1953 gestorben: Die CDU/CSU steigerte sich von 31, o Prozent in 1949 auf 45,2 Prozent, die SPD erreichte 28,8 Prozent und verlor 0,4 Prozentpunkte.

\subsubsection{Konflikte in der Einheitsgewerkschaft}

Zwischen den Christlich-Sozialen und den Sozialdemokraten im Führungsgremium der GTB zeigten sich Mitte der 1950er Jahre zunehmend Meinungsverschiedenheiten. Die SPD-Mitglieder sahen keine Veranlassung, ihre Positionen und Strategien den neuen Verhältnissen anzupassen, obwohl die Hoffnung, einen wirtschaftsdemokratischen Ansatz durchzusetzen, schon 1952 mit der

61 Zonenausschuß der CDU für die britische Zone (1947): Ahlener Programm, Ahlen/Westfalen, 3. Februar 1947. 
Verabschiedung des Betriebsverfassungsgesetzes einen deutlichen Rückschlag erhalten hatten. Die sich etablierende wirtschaftliche Ordnung wurde programmatisch ignoriert, die Vorstellung einer wirtschaftlichen Neuordnung auch ohne realistische Aussicht auf Umsetzung weiter vertreten.

Eine Ausnahme unter den Sozialdemokraten bildete Karl Buschmann, der 1951 im Alter von 37 Jahren als jüngstes Mitglied in den GHV gewählt wurde. Der Gewerkschafter war 1914 geboren und hatte vor 1933 keine gewerkschaftlichen Funktionen inne. Buschmann entwickelte sich zunehmend zum Gegenspieler des Vorsitzenden Werner Bock. Diese Konstellation spiegelt die Auseinandersetzungen jener Zeit in der allgemeinen Gewerkschaftsbewegung wider:Vertreter:innen des sozialen Katholizismus und Sozialdemokrat:innen, die ihre Partei von der Klassenpartei zur Volkspartei wandeln wollten, standen den "Traditionalisten« gegenüber, die die 1949 beschlossenen Grundsätze für eine künftige Wirtschaftsordnung hochhielten.

Teile der katholischen Arbeitnehmerbewegung begannen vor dem Hintergrund der aus ihrer Sicht zu einseitig agierenden Einheitsgewerkschaften eine Diskussion um eine eigenständige christliche Gewerkschaftsgründung. ${ }^{62}$ Aber auch konservative Kreise, die an der Schwächung der Einheitsgewerkschaften interessiert waren, drängten in diese Richtung. Innerhalb des Deutschen Gewerkschaftsbundes (DGB) entzündeten sich die Konflikte um die Einheitsgewerkschaft besonders an der Person von Viktor Agartz (siehe Kapitel 3.1.6). Dessen im Oktober 1954 auf dem DGB-Bundeskongress umjubelten Beitrag nannte selbst der GTB-Christdemokrat Tacke seinerzeit

»eine kristallklare Analyse der besehenden Gesellschaft- und Wirtschaftsordnung in der BRD und als eine schonungslose Abrechnung mit ihren sich sozial tarnenden Herrschaftskräften ${ }^{63}$

Wenige Wochen später waren von christlich-sozialen Gewerkschaftern andere Stimmen zu hören. Ein Cheftheoretiker, der vor der Illusion der Sozialpartnerschaft warnte und auf marxistischer Grundlage arbeite, sei für die Einheitsgewerkschaft nicht tragbar, hieß es. Insbesondere Oswald von Nell-Breuning, der Spiritus Rector der christlichen Soziallehre, warf Agartz im Januar 1955 vor, den DGB »in einen Irrgarten staats-, gesellschafts- und wirtschaftspolitischer Wahnvorstellungen« zu führen. Er hielt dem Leiter des Wirtschaftswissenschaftlichen Instituts des DGB (WWI) klassenkämpferischen Radikalismus und Verantwor-

62 So ein Bericht von Bernhard Tacke, dem zufolge die Katholische Arbeitnehmer-Bewegung (KAB) für die Bildung eines christlichen Gewerkschaftsbundes plädierte; GTB: Protokoll der Beiratssitzung vom 2./3. Juni 1955, AdsD 5/GTBA0301001. 
tungslosigkeit vor und warnte vor einer Spaltung des DGB.$^{64}$ Nell-Breuning war ein Verfechter der Einheitsgewerkschaft und wollte den sozialen Katholizismus innerhalb des DGB halten.

Von den Mitgliedsgewerkschaften des DGB hielten besonders die IG Bau-Steine-Erden und die IG Bergbau und Energie eine programmatische Anpassung an die bestehenden Verhältnisse der sozialen Marktwirtschaft für unvermeidlich. Agartz hielt an den Neuordnungsvorstellungen fest, die eine stärker gemeinwirtschaftliche Ausrichtung der Wirtschaft einschließlich vermehrter planerischer Elemente beinhalteten, ${ }^{65}$ und verlor schließlich die politischen Positionskämpfe. Er wurde im Oktober 1955 beurlaubt und trat zum Jahresende als Leiter des WWI zurück.

Im GTB-Führungsgremium bekannten sich die Christlich-Sozialen Tacke und Karpf klar zum Konzept der Einheitsgewerkschaft. Gleichzeitig kritisierte insbesondere Tacke sowohl die DGB-Führung als auch Bock. Er stellte die Frage, warum sich die Gewerkschaften gesellschaftspolitisch mit den umstrittenen Fragen der Westintegration und Remilitarisierung beschäftigten; CDU-orientierte Arbeitnehmer verstünden das nicht. Tacke forderte weltanschauliche Toleranz, damit sich alle in der Einheitsgewerkschaft wiederfinden könnten. ${ }^{66}$ Bock konterte:

»Man kann nur das verteidigen, was verteidigungswert ist. Unsere gesamte Gesellschaftsform ist auf den reinen Kapitalismus eingestellt. [...] Was wir hier haben ist nicht verteidigungswert. Dagegen gibt es im Osten manches was wir wollen. « ${ }^{67}$

Im Hinblick auf die engere gewerkschaftliche Politik stellte Tacke die Frage, warum nur Opposition gelebt werde:

»Das führt dazu, daß selbst tatsächliche Erfolge und Fortschritte negiert werden und somit die Erfolglosigkeit der eigenen Arbeit immer wieder hervorgehoben wird. Auf die Dauer muss das dazu führen, Zweifel an der Richtigkeit des eigenen Wollens zu bekommen. ${ }^{68}$

Tacke stellte infrage, dass die Gewerkschaften

»so tun, als ob hier alles noch so wäre wie ehedem und den einstmals gegebenen Gegensatz zwischen Arbeitgebern und Arbeitnehmern noch 10oprozentig als

64 Nach Jünke (2014): Das dritte Leben des Viktor Agartz.

65 Krämer (1995): Viktor Agartz: Vom Cheftheoretiker zur »Persona non grata«, S. 314.

66 CTB: Protokoll der Beiratssitzung vom 2./3. Juni 1955, AdsD 5/GTBA0301001.

67 CTB: Protokoll der Beiratssitzung vom 11. Februar 1955, AdsD 5/CTBA0301001.

68 GTB: Protokoll der Beiratssitzung vom 11. Februar 1955, AdsD 5/CTBA0301001. 
bestehend betrachten, oder ob wir das in vielen Betrieben irgendwie geänderte Verhältnis erkennen und unsere gewerkschaftliche Aufgabe daran auszurichten versuchen. ${ }^{69}$

Der profilierte Bernhard Tacke wurde 1956 in den DGB-Bundesvorstand gewählt, Hugo Karpf übernahm das Amt des Stellvertretenden GTB-Vorsitzenden. Den vakanten Platz im GHV nahm Martin Heiß ein.

Bei den Bundestagswahlen 1957 konnte die CDU/CSU ihr gutes Wahlergebnis von 1953 noch ausbauen. In der Sozialdemokratie bekam die Diskussion um programmatische Veränderungen eine neue Dynamik und führte 1959 schließlich zur Verabschiedung des Godesberger Programms. Mit diesem Grundsatzprogramm stellte sich die SPD neu auf, machte ihren Frieden mit dem marktwirtschaftlichen System und bekannte sich politisch zu Westbindung und Verteidigungspolitik. Anstatt Arbeiterpartei mit klassenkämpferischer Rhetorik wollte man nun Volkspartei sein. $^{70}$

Werner Bock, der Vorsitzende der GTB, stand der Reformdiskussion im DGB und der Entwicklung der SPD als Sozialdemokrat ebenso kritisch gegenüber wie seine Vorstandskollegen Paul Trost und Fritz Knepper. Auch Liesel Kipp-Kaule, die seit 1949 nicht nur dem GHV, sondern zusätzlich zu ihrer Gewerkschaftstätigkeit auch dem Bundestag angehörte, zählte zu den »Traditionalisten« (oder auch "Linken«), die sich auf eine klare Mehrheit im Hauptvorstand und im Beirat stützen konnten. Doch die Erfolglosigkeit beim Ringen um radikale Veränderungen des Wirtschaftssystems stärkte die Stimmen derer, die diesem Kurs gegenüber kritisch eingestellt waren.

1959 stand ein weiterer Personalwechsel an. Hugo Karpf schied altersbedingt aus, der Christdemokrat Karl Dörpinghaus wurde Stellvertretender Vorsitzender. Damit blieb der nirgends festgeschriebene, aber 1949 gefundene Proporz zwischen Sozialdemokraten und Christlich-Sozialen von fünf zu zwei GHV-Mitgliedern unangefochten.

\subsubsection{Oppositionelle Gemeinschaft der »Christen« und "Godesberger"}

Während die Mehrheit in den Führungsgremien weiter den traditionellen Kurs verfolgte, arbeiteten vor allem jüngere Gewerkschafter an einem Kurswechsel. Von Baden-Württemberg aus bildeten die »Godesberger Sozialdemokraten« in der GTB eine Allianz mit den christlich-sozialen Kolleg:innen von der Christlich-

69 CTB: Protokoll der Beiratssitzung vom 11. Februar 1955, AdsD 5/CTBA0301001.

70 Mit dem Godesberger Programm vollzog die SPD den programmatischen Wandel von einer sozialistischen Arbeiterpartei zu einer Volkspartei, wie das Bekenntnis zu Marktwirtschaft und Landesverteidigung zeigt. 
Demokratischen Arbeitnehmerschaft (CDA). Ihr Ziel war, mittelfristig eine Alternative zur Mehrheit der »Traditionalisten« bei den GHV-Mitgliedern zu etablieren und damit den traditionellen Linkskurs der GTB zu korrigieren.

Die »Reformer« in der GTB verwiesen darauf, dass die einseitige Ausrichtung der Einheitsgewerkschaft politisch eher konservativ eingestellte Arbeitnehmer:innen von einer Mitgliedschaft abschrecke, denn seit 1955 waren die christlichen Gewerkschaften wieder in einem Dachverband vereinigt und warben aggressiv um die Beschäftigten, die sich CDU und CSU verbunden fühlten. Viele durch die Textilindustrie geprägte Regionen Westdeutschlands waren politisch konservativ und katholisch geprägt. Vor 1933 hatten die christlichen Gewerkschaften hier starke Positionen, weshalb diese Regionen nun als potenziell anfällig für eine Abspaltung galten.

Die zunächst kleine oppositionelle politische Minderheit ging strategisch planvoll vor. Galionsfigur derer, die sich als »Reformer« oder »Modernisierer« sahen, war Karl Buschmann, der in der Organisation beliebt war. Der Tarifpolitiker, stark von den Erfahrungen der US-Gewerkschaften geprägt, arbeitete an inhaltlichen Veränderungen, für deren Umsetzung ein entspanntes Verhältnis zu den Arbeitgebern hilfreich, wenn nicht sogar notwendig war. Die offenen Auseinandersetzungen mit den "Traditionellen« bestritten die jungen Baden-Württemberger Bezirkssekretäre Willi Werner (später Bezirksleiter) und Berthold Keller (später GTB-Vorsitzender).

Das gezielte Vorgehen wurde schon auf dem Gewerkschaftstag 1955 sichtbar und in den Folgejahren konsequent fortgesetzt. Die »Reformer « setzten durch die strategische Besetzung von Gewerkschaftstags-Mandaten und abgestimmte kritische Redebeiträge Nadelstiche gegen die GHV-Mehrheit. Werner Bock war als "Gründungsvater« der GTB schwer angreifbar, auch wenn er in den Augen der Kritiker als personifizierter Ausdruck der Erstarrung der Gewerkschaftsbewegung galt. Anstatt offensiv für ein alternatives Programm zu streiten, versuchten die »Reformer «, die Autorität der »Traditionalisten« zu demontieren. Sie kritisierten das Handeln »im alten Stil«, griffen populäre Themen auf, die vermeintlich zu wenig vorangetrieben wurden und hakten ein, wenn zentrale Entscheidungen oder das Handeln des GHV von vielen zumindest als unglücklich empfunden wurden.

So präsentierte Berthold Keller auf dem Gewerkschaftstag 1957 eine breite Palette an Themen. Er kritisierte den Vorstand wegen unabgestimmten Vorgehens in einer Tariffrage, die an der Basis für Unmut gesorgt hatte, und warnte vor einer verfehlten Personalpolitik mit falschen Schwerpunkten. Darüber hinaus gehe man nicht mit der Zeit, was die Mitgliederbetreuung betraf: 
»Man kann nicht mehr nach den Maßstäben operieren, die vielleicht noch vor 20 oder 30 ]ahren in Ordnung waren. [...] Die Zeit wandelt sich und da muss man neue Methoden anwenden und Konsequenzen ziehen. « ${ }^{71}$

Geschickt wurde der Unmut aufgegriffen, der sich aus verschiedenen Gründen bildete. Beispielsweise sah Willi Werner mit dem Vorschlag des Vorstandes, den Gewerkschaftstag nur noch alle drei statt alle zwei Jahre tagen zu lassen, das Gleichgewicht zwischen Legislative und Exekutive grundsätzlich gestört. Der entsprechende Antrag erhielt daraufhin nicht die erforderliche Mehrheit. ${ }^{72}$ Zwei Jahre später unterstellten Keller und Werner dem Vorstand bereits zu Beginn des Kongresses in einer von ihnen ausgelösten Debatte zur Geschäftsordnung undemokratische Verhaltensweisen. Sie forderten mehr Rechte für die Delegierten und eine Beschneidung der Macht der GHV-Mitglieder und Bezirksleiter. ${ }^{73}$ Nicht immer setzten sie sich mit der Kritik am Vorstand und dessen Arbeit durch, doch sie ließen sich von Rückschlägen nicht beeindrucken und arbeiteten weiter an der Destabilisierung der GHV-Mehrheit. In Kommissionen und der neu gegründeten Personalvertretung platzierten sie ihre Anhänger:innen.

Die »Reformer hatten einen objektiven Schwachpunkt ausgemacht. Die großen Streiks der 1950er Jahre (siehe Kapitel 3.1.3 und 3.1.5) hatten die GTB an den Rand ihrer finanziellen Möglichkeiten gebracht, da es nicht gelungen war, den gewerkschaftlichen Organisationsgrad zu erhöhen. Vielmehr blieb die Mitgliederentwicklung in der Ära Werner Bock deutlich hinter der Beschäftigtenentwicklung zurück; der gewerkschaftliche Organisationsgrad verringerte sich in dieser Zeit von 55 Prozent auf 38 Prozent. Dies entsprach zwar dem gesamtgesellschaftlichen Trend, aber im Textil-Bekleidungs-Bereich fiel der Rückgang deutlicher aus als in anderen Branchen. Auf die gesamte Bundesrepublik bezogen sank in diesem Zeitraum das Verhältnis der Mitglieder in den DGB-Gewerkschaften zur Gesamtzahl der Beschäftigten von 43 Prozent auf ebenfalls 38 Prozent. ${ }^{74}$

Die Wirtschaft bewegte sich auf Vollbeschäftigung zu, die GTB wie auch die anderen Gewerkschaften erzielten achtbare tarifpolitische Erfolge - und dennoch verweigerte ein größer werdender Anteil der Beschäftigten die Mitgliedschaft. Durch den Ausbau des Sozialstaats verloren die gewerkschaftlichen Unterstützungsleistungen an Bedeutung, aufgrund des zunehmenden Arbeitskräftemangels trat die Schutzfunktion der Gewerkschaften in den Hintergrund. Entscheidend aber war, dass die Arbeitgeber die mit den Gewerkschaften ausgehandelten

71 GTB: Protokoll des 5. Ordentlichen Cewerkschaftstages, 2.-5. Juli 1957 in Kassel, S. 69.

72 GTB: Protokoll des 5. Ordentlichen Gewerkschaftstages, 2.-5. Juli 1957 in Kassel, S. 157.

73 GTB: Protokoll des 6. Ordentlichen Gewerkschaftstages, 24.-29. August 1959 in Hamburg, S. $46 f f$.

74 Greef (2014): Gewerkschaften im Spiegel von Zahlen, Daten und Fakten, S. 703. 
Tarifleistungen auch Unorganisierten gewährten, obwohl sie dazu nur gegenüber den Gewerkschaftsmitgliedern verpflichtet waren.

Die »Reformer« hatten das Ziel, die GTB durch eine stärkere Etablierung als Akteurin in den Branchen und durch ein entspanntes Verhältnis zu den Arbeitgebern für die Beschäftigten attraktiver zu machen. Den Arbeitgebern und ihren Verbänden stellten sie eine Sozialpartnerschaft in Aussicht, wenn sie die veränderte Rolle und Politik der GTB stärker würdigen und ihr Handeln anerkennen würden.

\subsubsection{Kommunistische Unterwanderung?}

Die politischen Auseinandersetzungen spielten sich vor dem Hintergrund der Systemkonkurrenz zwischen den »realsozialistischen« Staaten des Ostblocks und den marktwirtschaftlich-kapitalistischen der westlichen Welt ab. Vermeintlich »linkes« Handeln in der Bundesrepublik war den Umarmungsversuchen der DDR ausgesetzt. Vor allem die Streiks der 1950er Jahren wollten kommunistische Agitator:innen aus der DDR in ihrem Sinne nutzen. Der GTB-Geschäftsbericht 1959/1960 beklagt die Politik aus der Sowjetzone, »die Gewerkschaftsbewegung zu unterwandern «. ${ }^{75}$ Die Versuche, Mitglieder durch Flugblätter und Postsendungen zu beeinflussen, seien jedoch erfolglos geblieben.

Es gab aber darüber hinausgehende Aktivitäten, die keine Erwähnung im Geschäftsbericht fanden. Während der Textilkrise 1958 empfing Fritz Knepper, Mitglied des Geschäftsführenden Hauptvorstandes der GTB, am 6. Oktober in seinem Düsseldorfer Büro eine Delegation der DDR-Gewerkschaft Textil-Bekleidung-Leder (IG TeBeLe) samt der Vorsitzenden und ihrem Stellvertreter. In Ostberlin hatte zuvor eine Konferenz mit 120 Teilnehmern aus der Bundesrepublik stattgefunden, die über die schwierige wirtschaftliche Situation in der westdeutschen Textilindustrie beriet. So titelte die GTB-Zeitung mit »Notschrei der Kurzarbeiter « und schrieb angesichts der andauernden Kurzarbeit und des geringen Lohnersatzes von einem echten Notstand, der in den westfälischen Textilorten Rheine und Schüttorf erhebliche Auswirkungen auf die regionale Wirtschaft habe. ${ }^{76}$

Die Ostdelegation unterbreitete der GTB als Ergebnis der Konferenz das Hilfsangebot, die Beschäftigung in der westdeutschen Textilindustrie durch einen 90-Millionen-DM-Auftrag zu stabilisieren. Fritz Knepper und Paul Trost setzten sich im GTB-Hauptvorstand für eine Prüfung dieses Angebotes ein. ${ }^{77}$ Bereits 1956

75 GTB: Geschäftsbericht 1959-1960 des Hauptvorstandes, S. 41.

76 textil-bekleidung, Ausgabe 12/1958, S. 3.

77 GTB: Protokoll der Sitzung des Geschäftsführenden Hauptvorstandes vom 17. Dezember 1958, AdsD 5/GTBA010007. 
hatte Trost den Zweiten Vorsitzenden der Ostgewerkschaft sowohl bei sich zu Hause als auch im Büro empfangen. Die Kontakte von Knepper und Trost wurden vom GTB-Justiziar Herbert Weder 1959 öffentlich gemacht, einem Renegaten, der nach eigenen Angaben von 1955 bis 1957 intensive Kontakte zum FDGB der DDR aufgebaut hatte. Er unterstellte eine systematische Unterwanderung durch linientreue Kommunisten mit den beiden GHV-Mitgliedern an der Spitze. ${ }^{78}$

Um die Vorwürfe zu prüfen, beurlaubte der GTB-Beirat Knepper und Trost, aber auch Weder selbst, und setzte eine Untersuchungskommission ein. Bei der Besetzung dieser Kommission setzten sich in Kampfabstimmungen die »Traditionalisten durch. $^{79}$ Der DGB-Bundesvorstand zeigte sich beunruhigt und lud den GHV und die Untersuchungskommission für Anfang Mai 1959 zur Berichterstattung vor - die jedoch ein Treffen zu diesem Zeitpunkt ablehnten, was beim DGB Befremden auslöste. ${ }^{80}$ Nach Presseberichten sah man in der DGB-Spitze die Gewerkschaft Holz und Kunststoff und die GTB als besonders anfällig für kommunistische Unterwanderung.

»Der Spiegel« vermeldete die Befürchtung, Knepper und Trost arbeiteten daran, die GTB kommunistisch auszurichten. Ziel sei das Ausscheren aus der kritischen DGB-Linie gegenüber dem SED-hörigen FDGB und die Aufnahme offizieller Beziehungen. ${ }^{81}$ Die »Frankfurter Neue Presse « unterstellte Werner Bock einen regelmäßigen brieflichen und persönlichen Kontakt mit dem Zweiten Vorsitzenden der IG TeBeLe, Otto Lehmann. ${ }^{82}$ Die "Allgemeine Sonntagszeitung«, die man durchaus als christlich-konservatives Kampfblatt bezeichnen kann, schrieb im April 1959 unter der Überschrift »Von Leuten, die im Trüben fischen« über die "peinlichen und sorgsam geheim gehaltenen Querverbindungen zwischen den maßgeblichen Leuten [der GTB] und Spitzenfunktionären der entsprechenden KP-Organisation in der Zone«. ${ }^{83}$

Die interne GTB-Untersuchungskommission kam zu dem Schluss, die Ostkontakte von Trost und Knepper seien keine Verschwörung mit dem Ziel gewesen, die GTB oder gar den Staat »zu verändern oder zu zerstören «, woraufhin der Beirat die Beurlaubung der beiden GHV-Mitglieder im Juni 1959 wieder aufhob. ${ }^{84}$ Der Justiziar Weder wurde entlassen, vor dem Arbeitsgericht verglich man sich mit

78 Anonym (o. .).): Die Genossen unter sich, S. 15.

79 GTB: Protokoll der Beiratssitzung vom 12./13. März 1959, AdsD 5/CTBA0301002.

80 GTB: Protokoll der Sitzung des Geschäftsführenden Hauptvorstandes vom 29. April 1959, AdsD 5/GTBA010007.

81 Der Spiegel (1959): Ostkontakte-Anruf vom Politruk, in: Ausgabe 15 vom 8.4.1959, S. 26-28.

82 Frankfurter Neue Presse (1959): DGB-Funktionär setzt sich ab, in: Ausgabe vom 23.5.1959.

83 Lomba (1959): Von Leuten, die im Trüben fischen, in: Allgemeine Sonntagszeitung, Nr. 16 vom 19.4.1959.

84 GTB: Protokoll der Beiratssitzung vom 18./19. Juni 1959, AdsD 5/CTBA0301002. 
einer Abfindungssumme von 12.000 DM. ${ }^{85}$ Ohne Einschränkung wurde nun allen Gewerkschaftsmitgliedern der Kontakt mit Funktionär:innen oder Beauftragten der DDR untersagt. Diese Abgrenzung wurde dadurch unterstrichen, dass zwei GTB-Funktionäre der unteren Ebene mit regelmäßigen DDR-Kontakten und öffentlichen Einlassungen im Sinne des ostdeutschen Staates entlassen wurden, und zwar der Geschäftsführer der Verwaltungsstelle Düsseldorf und ein Gewerkschaftssekretär der Verwaltungsstelle Augsburg. ${ }^{86}$

Auf dem Gewerkschaftstag 1959 verweigerten Bock, wie Trost und Knepper dazu weitere Auskünfte. Knepper erklärte, auch im Namen von Trost:

»[...] daß wir beide zusammen oder jeder für sich mit Vertretern des FDCB oder sonstigen Stellen von drüben konspirative Gespräche oder Verbindungen gehabt haben, nicht anderes als Lüge sind. $\aleph^{87}$

Als der Delegierte Ernst Wenzel nachhakte:

»[...] was haben Sie denn mit diesen Funktionären besprochen? [...] Um über das Wetter zu sprechen, wird es, so glaube ich, niemandem einfallen, von der Ostzone nach Düsseldorf zu kommen«,

erntete er »lebhaften Widerspruch, Protestrufe, Pfui-Rufe«. ${ }^{88}$ Die große Mehrheit der Delegierten sah in den Vorwürfen eine Verschwörung von rechten Gewerkschaftsfeinden, auf deren Niveau man sich zumindest zu diesem Zeitpunkt nicht einlassen wollte. Trost und Knepper wurden mit 75 Prozent und 72 Prozent der abgegebenen Stimmen in ihren Ämtern bestätigt und erhielten damit deutlich bessere Ergebnisse als die Christlich-Sozialen Dörpinghaus und Heiß.

Doch damit hatte die Geschichte noch nicht ihr Ende gefunden. Nach Anzeige von Weder bei der Bundesanwaltschaft erhob diese Anklage gegen Paul Trost und Fritz Knepper wegen des Verdachts auf Hochverrat, was die Presse und den DGBBundesvorstand 1961 nochmals beschäftigte. Dies war jedoch für die GTB und die beiden Funktionäre kein Anlass, ihre Ämter erneut ruhen zu lassen, was Karl Buschmann im Hauptvorstand kritisierte. Doch die »Traditionalisten « hatten die Mehrheit im Hauptvorstand, so dass dieser in einer Presseerklärung betonte, in keiner Form in die Ermittlungen eingreifen zu wollen, und auf das Ergebnis der

85 GTB: Protokoll der Sitzung des Ceschäftsführenden Hauptvorstandes vom 12. März 1961, AdsD 5/CTBA0220067.

86 Anonym (o.J.): Die Genossen unter sich, S. 18-21.

87 CTB: Protokoll des 6. Ordentlichen Cewerkschaftstages, 24.-29. August 1959 in Hamburg, S. 214.

88 GTB: Protokoll des 6. Ordentlichen Cewerkschaftstages, 24.-29. August 1959 in Hamburg, S. 214. 
internen Untersuchung verwies ${ }^{89}$ Im März 1962 entschied der Staatsschutzsenat des Bundesgerichtshofs, das Hauptverfahren nicht zu eröffnen und Trost und Knepper außer Verfolgung zu setzen..$^{90}$

\subsubsection{Die "Reformer" setzen sich durch}

Das Jahr 1961 stand im Zeichen der Richtungsentscheidungen: Sollten die Verfechter:innen einer grundsätzlich systemkritischen und auf konfrontatives Austragen von Konflikten mit den Arbeitgebern gerichteten Strategie weiter den Kurs an der Spitze bestimmen oder diejenigen, die Formen und Inhalte der GTB für anpassungsbedürftig hielten? Auf dem für Oktober des Jahres anberaumten 7. Ordentlichen Gewerkschaftstag schied der bisherige Stellvertretende Vorsitzende Karl Dörpinghaus aus Altersgründen aus. Bislang war auf diese Position immer ein CDU- bzw. CSU-Mitglied gewählt worden. Dörpinghaus verzichtete jedoch im Namen der Christlich-Sozialen und sicherlich in Abstimmung mit den moderaten Sozialdemokraten auf die Besetzung dieser Position mit einem Kandidaten aus dem christlich-sozialen Lager. ${ }^{91}$ Damit stand fest, dass es bei der Wahl seines Nachfolgers um die Aufstellung für die zwei Jahre später zu wählende Nachfolge von Werner Bock ging. Würde ein »Modernisierer« diesen Platz einnehmen oder die »Traditionalisten« ihre Position verteidigen?

Es wurde der emotionalste Kongress in der GTB-Geschichte. Erstmals nach 1949 wurden die politischen Konflikte offen über Kampfkandidaturen ausgetragen. Beide Lager versuchten, vermeintlich unentschiedene Delegierte auf ihre Seite zu ziehen. Diese innergewerkschaftliche Rechts-links-Auseinandersetzung fand auch vor dem Hintergrund der deutschlandpolitischen Entwicklungen statt. Gesellschaftlich hatten schon der 17. Juni 1953 und der Ungarnaufstand 1956 die antikommunistische Stimmung befördert, nun erhöhte die im August 1961 errichtete Berliner Mauer den Konformitätsdruck erheblich. Die Spaltung Deutschlands schien auf drastische Weise besiegelt. Selbstverständlich geißelten das auch die links stehenden Sozialdemokraten.

„Über Grenzpfähle und Todesstreifen, über Stacheldraht und Eisernen Vorhang « grüßte Werner Bock die Kolleg:innen in Ostdeutschland, »die in den letzten Wochen und Monaten die Unfreiheit und die Knute des Zwangs aufs härteste erle-

89 CTB: Protokoll der Sitzung des Ceschäftsführenden Hauptvorstandes vom 12. Mai 1961, AdsD 5/ CTBA0220067.

90 CTB: Protokoll der Beiratssitzung vom 3./4. Mai 1962, AdsD 5/GTBA0301002.

91 CTB: Protokoll der Sitzung des Geschäftsführenden Hauptvorstandes vom 12. Mai 1961, AdsD 5/ CTBA0220067. 
ben mussten «. ${ }^{92}$ Jedoch waren die unbestreitbaren Kontakte zu Funktionär:innen aus dem FDGB sicher noch in manchem Hinterkopf.

In den meisten Diskussionsbeiträgen ging es vorder- oder hintergründig um die zukünftige Ausrichtung der Organisation und die anstehende PersonalVorentscheidung für die Bock-Nachfolge. Je nach Standpunkt wurde die Arbeit des einen gelobt, die Defizite der anderen unterstrichen. Linus Leusbrock, GTBGeschäftsführer aus Borghorst und CDU-Mitglied, eröffnete die Debatte zum Geschäftsbericht mit der Kritik, viele Gewerkschaftsaustritte im Münsterland hätten zum Grund, »daß man mit dem Kurs der Gewerkschaftsbewegung nicht zufrieden ist«. Er forderte, »diesen Kurs wieder dahin zu lenken, daß alle ihre Heimat in unserer Gewerkschaftsbewegung finden können..$^{93}$

Werner Arend, Geschäftsführer der GTB-Verwaltungsstelle Delmenhorst und Sozialdemokrat aus dem traditionellen Lager konterte, Leusbrock solle konkret sagen, mit welchen wirtschafts- und gesellschaftspolitischen Vorstellungen er nicht einverstanden sei. ${ }^{94}$ Auf der Seite der »Traditionalisten « verwies man darauf, dass die Unternehmer keinesfalls ihren Frieden mit den Gewerkschaften gemacht hätten. Deshalb verwahre man sich explizit dagegen,

»die Grundsätze, die damals auf dem Münchner Cründungskongress des Deutschen Cewerkschaftsbundes aufgestellt worden sind, heute als eine Jugendsünde auszulegen, so wie es verschiedene politische Parteien mit ihren Programmen machen ${ }^{95}$

Angesichts des Richtungsstreits waren weitere Kampfkandidaturen nicht ausgeschlossen, weshalb die Christlich-Sozialen angesichts ihrer stets schwächeren Ergebnisse die Gefahr sahen, dass ein Vertreter aus ihren Reihen bei der Wahl durchfallen könnte. Deshalb betonte die »Reformer«-Seite, man müsse die christdemokratisch orientierten Mitglieder und Funktionär:innen der GTB »unter echter Gleichberechtigung « beteiligen. Die GTB-Führung solle die »parteipolitische und religiöse Neutralität« wahren, vor allem aber nicht an den zwei »schwarzen« GHV-Mandaten rütteln.

Exemplarisch für die Stimmung und die Unsicherheit war ein Initiativantrag, wonach vor der Wahl die Zusammensetzung des GHV mit fünf Sozialdemokrat:innen und zwei Christlich-Sozialen festgeschrieben werden sollte, um deren Niederlage bei den sich abzeichnenden Kandidaturen von vornherein aus-

92 GTB: Protokoll des 7. Ordentlichen Cewerkschaftstages, 2.-6. Oktober 1961 in Nürnberg, S. 12.

93 GTB: Protokoll des 7. Ordentlichen Gewerkschaftstages, 2.-6. Oktober 1961 in Nürnberg, S. 105.

94 GTB: Protokoll des 7. Ordentlichen Gewerkschaftstages, 2.-6. Oktober 1961 in Nürnberg, S. 108.

95 Delegierter Willy Lindner, zit. nach: GTB: Protokoll des 7. Ordentlichen Gewerkschaftstages, 2.-6. Oktober 1961 in Nürnberg, S. 161. 
zuschließen. Ein Teil der christlich-sozialen Funktionär:innen warnte vor den Werbeversuchen des Christlichen Gewerkschaftsbundes vor allem in den katholisch geprägten Textilregionen und befürchtete den »Todesstoß für die Einheitsgewerkschaft", sollten bei den erwarteten Kampfkandidaturen christlich-soziale Vorstandsmitglieder unterliegen. Andere kritisierten einen möglichen "Naturschutzpark" für die "Schwarzen«, unabhängig von der Bewertung ihrer Arbeit. Für den fixierten Minderheitenschutz sprach sich auch ein Teil der »reformorientierten« Sozialdemokraten aus.

Die von gegenseitigen Vorwürfen geprägte Debatte wurde schließlich vom Vorsitzenden Werner Bock mit den Worten »Genug des grausamen Spiels!« beendet. Auf seinen Vorschlag hin wurde der Antrag auf den festgeschriebenen Minderheitenschutz nach einer Unterbrechung der Konferenz zurückgezogen, weil man »nicht etwas tun [kann], das in unserer deutschen Gewerkschaftsbewegung nicht gang und gäbe ist $\ll{ }^{96}$ Die Autorität des Vorsitzenden hatte noch einmal gewirkt.

Der Ausgang des Machtkampfes war zu Beginn alles andere als eindeutig. Die "Traditionalisten « hatten sich im Hauptvorstand und Beirat bisher immer durchgesetzt, und noch vor den Wahlen hatte der Gewerkschaftstag in deren Sinne beschlossen, die Wörter "Sozialpartnerschaft" und "Tarifpartner" in Gewerkschaftspresse, Gewerkschaftsversammlungen und Verhandlungen nicht mehr zu verwenden.${ }^{97}$ Auf der anderen Seite war der »Reformer« Karl Buschmann, der zur Wahl antrat, als Tarifpolitiker profiliert und in der Organisation beliebt, was bisher immer durch gute Wahlergebnisse bestätigt worden war. Buschmann nutzte seinen mündlichen Geschäftsbericht für eine kämpferische Rede, verwies auf die hohen Reallohnsteigerungen der letzten beiden Jahre, unterstrich das Zukunftsthema Rationalisierungsschutz und forderte tarifpolitische Exklusivleistungen für GTB-Mitglieder.

Die »Traditionalisten« schickten Georg Drescher, den niedersächsischen Bezirksleiter und Streikführer des Jahres 1958 ins Rennen, der vom Vorsitzenden Werner Bock favorisiert wurde. Die Debatte um den Minderheitenschutz fand vor dem Hintergrund statt, dass Bock eine weitergehende Strategie unterstellt wurde. Gerüchten zufolge sollte mit der Personalie Drescher ein Christlich-Sozialer "geopfert « werden, um die Anzahl der »Traditionalisten « im GHV von vier auf fünf zu erhöhen. Damit wäre der Kurs über das Ausscheiden Bocks hinaus gefestigt und die GTB im traditionellen Gewerkschaftslager um den IG Metall-Vorsitzenden Otto Brenner verankert worden.

Falls dieser Plan bestand, war er eine große Fehleinschätzung der Mehrheitsverhältnisse. Der moderate »Reformer« Buschmann gewann die Wahl zum

96 CTB: Protokoll des 7. Ordentlichen Cewerkschaftstages, 2.-6. Oktober 1961 in Nürnberg, S. 377.

97 GTB: Protokoll des 7. Ordentlichen Gewerkschaftstages, 2.-6. Oktober 1961 in Nürnberg, S. 454. 
Stellvertretenden Vorsitzenden eindeutig mit 148 Stimmen bei insgesamt 247 Delegierten. Er hatte alles auf eine Karte gesetzt und vor der Wahl erklärt, dass er im Falle einer Niederlage nicht mehr als »einfaches« Vorstandsmitglied kandidieren werde. Drescher erhielt nur 92 Stimmen und kandidierte anschließend als Beisitzer im GHV. Für die vier zu vergebenen Mandate bewarben sich die drei sozialdemokratischen »Traditionalisten « Drescher, Kipp-Kaule und Knepper sowie die CDU-Mitglieder Heiß und Bongartz und der sozialdemokratische »Reformer « Hoffmann. Alle drei Kandidaten des Reformerlagers setzen sich durch, Drescher unterlag mit 111 Stimmen erneut.

Das zweitschlechteste Ergebnis erzielte Fritz Knepper, Exponent der linken »Traditionalisten«, mit 121 Stimmen. Damit verfehlte er die Wiederwahl, weil der Christdemokrat Martin Heiß vier Stimmen mehr erhielt. Gründe für die Niederlage der Vertreter aus dem bislang führenden Lager waren neben dem verbreiteten Unbehagen über die nicht aufgearbeitete »DDR-Affäre« (siehe Kapitel 3.2.3) ein grundsätzlicher Stimmungsumschwung, an dem die »Godesberger Sozialdemokraten« gemeinsam mit den Christlich-Sozialen seit Jahren gearbeitet hatten und der durch den verschärften Ost-West-Konflikt begünstigt wurde.

Die Personalentscheidungen des Jahres 1961 sollten den Kurs der GTB für die nächsten dreißig Jahre bestimmen. Auf dem folgenden Kongress 1963, dem letzten mit Werner Bock als Vorsitzendem, zeigte sich die Mehrheit der »Reformer« erneut und bescherte den "Traditionalisten« eine weitere Niederlage. Die Wahl Buschmanns zum Vorsitzenden war nach der Vorentscheidung von 1961 unstrittig. Nun ging es um die repräsentative Vertretung der »Traditionalisten«. Zunächst wollte Drescher erneut als Stellvertretender Vorsitzender kandidieren, dann brachten die »Traditionalisten« Martin Lange, den Büroleiter von Werner Bock, als Konsenskandidaten für den GHV ins Spiel; im Gegenzug sollten die ChristlichSozialen wieder den Stellvertretenden Vorsitzenden stellen. Bei dieser Konstellation wäre das Kräfteverhältnis von drei »Traditionalisten« zu vier »Reformern« erhalten geblieben. Mit dieser starken Minderheit erhofften sich die "Traditionalisten« ein Korrektiv zur Fraktion des neuen Vorsitzenden Karl Buschmann zu werden.

Die »Reformer ließen sich darauf nicht ein und schickten Hermann Schumacher ins Rennen, der sich bereits als Personalvertreter in der Hauptvorstandsverwaltung mehrfach mit Bock angelegt hatte. Martin Lange kandidierte auch ohne Konsens. Die »Traditionalisten« hofften wie schon 1961 darauf, dass der wenig charismatische Christdemokrat Heiß bei der Blockwahl der vier weiteren GHVMitglieder durchfiel. Allerdings hatte der mittlerweile designierte neue Stellvertretende Vorsitzende Johann Bongartz erklärt, er werde die Wahl nicht annehmen, sollte sein christlich-sozialer Kollege durchfallen. ${ }^{98}$ 
Es kam erneut anders, als von den »Traditionalisten« geplant. Mit Schumacher und Lange wurden beide neuen Kandidaten gewählt. Dafür erreichte Liesel KippKaule, die einzige Frau im GHV der »Frauengewerkschaft « Textil-Bekleidung, die diesem seit 1949 angehörte, nur 111 von 232 Stimmen und musste das Gremium verlassen. Die »Reformer«, 1959 mit drei und 1961 mit vier Mitgliedern im GHV präsent, stellten nun fünf der sieben hauptamtlichen Vorstandsmitglieder. Auch von den acht Bezirksleitern wurden nur noch drei, nämlich die aus Niedersachsen, Minden-Lippe (Ostwestfalen) und Frankfurt (Hessen, Rheinland-Pfalz und Saarland), den »Traditionalisten« zugerechnet. Innerhalb von zwei Jahren hatte es an der Spitze der GTB eine komplette Wende gegeben. Die zuvor der kommunistischen Unterwanderung verdächtigte Gewerkschaft war nun eindeutig im Lager der gemäßigten Gewerkschaften um Georg Leber und die IG Bau-Steine-Erden verortet.

Anzumerken ist, dass die tarifpolitischen Erfolge, die sicherlich ein Baustein für Buschmanns Sieg waren, in erster Linie in den von den »Traditionalisten« dominierten Bezirken erstritten wurden. Diese bekamen verbal immer noch viel $\mathrm{Zu}$ stimmung. Ob der »Klassenkampf von oben« beklagt oder der Erfolg durch Kampf beschworen wurden, stets gab es viel Beifall. Andererseits waren die Realitäten in den Betrieben oftmals differenzierter und nur selten klassisch schwarz-weiß. Diese Diskrepanz zwischen der Rhetorik auf Gewerkschaftsveranstaltungen und der differenzierten Interessenvertretung in den Betrieben führte bei vielen Funktionär:innen, die in der Nachkriegszeit gewerkschaftlich sozialisiert waren, zu Unzufriedenheit. Die Rhetorik der Gewerkschafter aus der Weimarer Zeit wurde teilweise als überholt empfunden. Der Wunsch nach Verjüngung der Führung und Modernisierung der Gewerkschaft wurde lauter und schlug sich letztendlich in den Wahlergebnissen von 1961 und 1963 nieder.

Die Richtungsauseinandersetzungen traten in der GTB besonders zugespitzt zutage, aber die Frage nach der Ausrichtung innerhalb der mittlerweile etablierten Marktwirtschaft stellte sich in der gesamten Gewerkschaftsbewegung. Die Flügel wurden auf der einen Seite durch die IG Metall mit ihrem Vorsitzenden Otto Brenner und auf der anderen Seite durch die IG Bau-Steine-Erden mit Georg Leber an der Spitze repräsentiert. Nach Brenners Verständnis bestand die kapitalistische Klassengesellschaft weiterhin: Schlüsselindustrien sollten in Gemeineigentum überführt werden, an den Forderungen nach Mitbestimmung und volkswirtschaftlicher Planung wollte er festhalten. Für Leber ging es hingegen um Sozialpartnerschaft und konsequente Interessenvertretung unter den gegebenen Verhältnissen. ${ }^{99}$

Im »Godesberg des DGB «, dem DGB-Kongress von 1963, wurden diese Gegensätze nicht aufgelöst. Stattdessen wurde ein Programm beschlossen, das zwar die 
Wirtschafts- und Gesellschaftsstruktur weitgehend anerkannte, die sich in der Nachkriegszeit entwickelt hatte, aber zugleich betonte, dass die Entwicklung in der Bundesrepublik zu einer Wiederherstellung alter Besitz- und Machtverhältnisse geführt habe. Im Programm wurde beklagt, dass eine ständig fortschreitende Konzentration des Kapitals stattfinde, während die große Mehrheit der Bevölkerung von der Verfügungsgewalt über die Produktionsmittel ausgeschlossen bleibe. Deren Abhängigkeit von privater Wirtschaftsmacht sei nicht überwunden. ${ }^{100}$

Das Grundsatzprogramm von 1963 erschien vielen nicht aus einem Guss. Darin fand sich Kapitalismuskritik neben der Anerkennung der marktwirtschaftlichen Ordnung, ohne dass ein Bild für ein Gesellschaftsmodell nach gewerkschaftlichen Vorstellungen erkennbar wäre. Dieser Versuch der Modernisierung war nach einer relativ langen Zeit wirtschaftlicher Prosperität vom Optimismus über die Vermeidbarkeit kapitalistischer Krisen getragen. ${ }^{101}$ Allerdings konnte die GTB mit Blick auf »ihre« Branchen das Bild einer weitgehend krisenfreien Entwicklung der Wirtschaft nicht teilen. Der textile Motor stotterte immer wieder. Dennoch war die neue Mehrheit in der GTB-Führung von einem stetigen Wachstum und der Möglichkeit eines sozialen Ausgleichs mit den Unternehmern auf der Basis eines sachbezogenen Dialogs überzeugt.

\subsection{Die Ära Werner Bock: Tarifpolitische Erfolge und organisationspolitische Verluste}

Durch Übersiedlung von Unternehmen aus den ehemaligen Ostgebieten, dem Sudetenland und der sowjetischen Besatzungszone entstanden in der Bundesrepublik Deutschland neue Schwerpunkte der Textilindustrie. Um die Gewerkschaft flächendeckend präsent und handlungsfähig zu machen, musste der Hauptvorstand neue Verwaltungsstellen gründen. Es galt, Personal zu rekrutieren und die Verwaltungsstellen so zu gliedern, dass möglichst viele Mitglieder von hauptamtlichen Gewerkschaftssekretär:innen betreut werden konnten. Am Ende der Ära Bock hatte die GTB von ehemals 91 hauptamtlich und 135 nebenamtlich geführten Verwaltungsstellen nur noch 28 nebenamtlich geführte Verwaltungsstellen und 96 hauptamtlich besetzte Büros vor Ort. ${ }^{102}$ Diesen Konsolidierungsprozess gestaltete die Textilgewerkschaft bemerkenswert erfolgreich. In der Zeit von 1949

\footnotetext{
100 DGB: Protokoll des Außerordentlichen Bundeskongresses, 21./22. November 1963 in Düsseldorf, S. 452.

101 Vgl. hierzu Grebing (1990): Gewerkschaften: Bewegung oder Dienstleistungsorganisation 1955 bis 1965.

102 GTB: Geschäftsbericht 1961-1962 des Hauptvorstandes, S. 18.
} 
bis 1952 erhöhte sie ihren Mitgliederstand von 300.000 auf 430.000 und erreichte damit einen gewerkschaftlichen Organisationsgrad von über 50 Prozent. ${ }^{103}$

Parallel dazu musste die Tarifpolitik gestaltet werden. Das Warenangebot war nach der Währungsreform im Juni 1948 mit Einführung der D-Mark deutlich gestiegen, allerdings auch die Preise. Die Mitglieder erwarteten entsprechende Lohnerhöhungen. Der Vorsitzende Werner Bock definierte für die GTB die »vornehmste Aufgabe, daß der Textil- und Bekleidungsarbeiter nicht am Ende im Lohngruppenvergleich stehen bleibt. ${ }^{104}$ Die Tariflandschaft bei Textil und Bekleidung war anfangs stark zersplittert und musste zunächst konsolidiert werden. Größeren Tarifgebieten wie Westfalen standen viele lokal ausgerichtete Tarifgebiete gegenüber, die in größeren Einheiten zusammenzufassen waren.

Im Zeitraum 1950-1952 wurden gleichzeitig beachtliche Lohnerhöhungen durchgesetzt und Abwehrkämpfe gegen Lohnkürzungen durchgeführt. Erst mit dem Westfalenstreik 1953 (siehe Kapitel 3.1.3) wurde diese erste, noch ziemlich unkoordinierte Phase der Tarifpolitik abgeschlossen. Anschließend konnten Themen wie die inhaltliche Neuordnung von Tarifverträgen, die Verkürzung der regelmäßigen Wochenarbeitszeit und die Verlängerung des Jahresurlaubs sowie weitergehende Forderungen koordiniert bearbeitet werden.

Im ersten Nachkriegsjahrzehnt war das Verhältnis zu den Arbeitgeberverbänden und den Unternehmern von grundsätzlicher Skepsis geprägt. Werner Bock hatte als Geschäftsstellenleiter des DTAV erlebt, wie die Textilunternehmer zur Zeit der Weltwirtschaftskrise im März 1931 Lohnkürzungen erzwungen hatten. Wenige Wochen später hatten sie alle Tarifverträge gekündigt und eine abermalige Lohnkürzung, besser noch die Abschaffung der Tarifverträge gefordert. ${ }^{105} \mathrm{Wie}$ dramatisch die Lage der Beschäftigten damals war, wird durch die Telegramme deutlich, die die drei damaligen Textilgewerkschaften gemeinsam an Reichspräsident und Reichskanzler sandten. In ihrem Hilferuf schrieben sie:

»Der [...] Lohnabbau hat sich auf die Lebenshaltung der Textilarbeiterschaft, die zu den schlechtbezahltesten zu rechnen ist, verheerend ausgewirkt. Die bereits vorhandene Not ist ins unermessliche gesteigert worden. $\aleph^{106}$

Bock war überzeugt, dass die Arbeitgeber auch in der jungen Bundesrepublik jede Gelegenheit nutzen würden, Erreichtes wieder infrage zu stellen und möglichst

\footnotetext{
103 GTB: Protokoll des 3. Ordentlichen Cewerkschaftstages, 16.-19. Juni 1953 in Düsseldorf, S. 41.

104 GTB: Protokoll des 2. Ordentlichen Cewerkschaftstages, 30. Mai-2. Juni 1951 in Düsseldorf, S. 44.

105 Cewerkschaftszeitung, Ausgabe 38 vom 19. September 1931, S. 608.

106 Cewerkschaftszeitung, Ausgabe 42 vom 17. Oktober 1931, S. 686.
} 
zurückzudrehen. So rief er die Delegierten auf dem Gewerkschaftstag 1953 zum Widerstand auf:

"So groß auch unser Respekt vor den Gerichten und so tief gegründet unser Vertrauen auf die Gerechtigkeit auch sein mag, wir sollten nicht bei allen Willkürakten der Unternehmer, bei allen Gesetzesverletzungen unser einziges Heil in den Arbeitsgerichten sehen und suchen. Willkür und Rechtsbruch müssen von den Belegschaften blitzschnell und deutlich beantwortet werden. ${ }^{107}$

In den Tarifkämpfen sah der Gewerkschaftsvorsitzende auch ein Mittel zur Emanzipation der Arbeiterschaft. Jeder tarifpolitische Fortschritt sei gleichzeitig ein Schritt zur Freiheit und zur Menschenwürde. ${ }^{108}$ Werner Bock blieb während seiner gesamten Amtszeit der Marktwirtschaft gegenüber kritisch eingestellt und gab seine Hoffnung auf eine grundsätzlich demokratisierte Wirtschaft nicht auf. Er forderte echte Mitbestimmung der Betriebsräte in Wirtschaftsangelegenheiten und überbetriebliche Wirtschafts- und Sozialräte. Immer wieder kritisierte er in den Gremien »unsere Gesellschaftsform, die auf reinen Kapitalismus eingestellt ist ${ }^{109}$

Einen hohen Stellenwert maß Bock der internationalen Gewerkschaftsarbeit zu. Er führte die GTB in die Internationale der Textil- und Bekleidungsgewerkschaften zurück und wurde zu deren Vorstandsmitglied gewählt. Bock verfolgte eine Politik der Unterstützung der Gewerkschaften in den Entwicklungsländern, wo man sich bereits in den 1950er Jahren zunehmend auf die Textilproduktion spezialisierte. Er war davon überzeugt, dass mit der Bildung und Stärkung der Gewerkschaften in den Niedriglohnländern höhere Einkommen durchgesetzt werden könnten. So würden regionale Binnenmärkte geschaffen und die alleinige Ausrichtung auf den Export in die Industrieländer gebremst. Dieser Weg schien Bock gerechter und vielversprechender für die Arbeiter:innen in den Industrieländern und in den Entwicklungsländern als protektionistische Maßnahmen der Industrieländer. Über die Entwicklung der ausländischen Schwestergewerkschaften und ihre Kämpfe wurde regelmäßig und ausführlich in der Mitgliederzeitung berichtet.

Der in Thüringen aufgewachsene Bock litt unter der deutschen Teilung. Bis 1959 betonte er in jedem seiner Geschäftsberichte die Hoffnung auf die Wiedervereinigung. In der westdeutschen Wiederbewaffnung und Westbindung sah

107 GTB: Protokoll des 4. Ordentlichen Gewerkschaftstages, 4.-8. Juni 1955 in Frankfurt am Main, S. 69.

108 GTB: Protokoll des 8. Ordentlichen Gewerkschaftstages, 16.-20. September 1963 in Hannover, S. 10.

109 GTB: Niederschrift der Beiratssitzung vom 10./11.2.1955, AdsD 5/CTBA030001. 
er Hindernisse auf diesem Weg. Vor dem Hintergrund des Kalten Krieges und der unzweifelhaften Versuche der DDR, Einfluss auf die westdeutsche Arbeiterbewegung auszuüben, waren Kontakte nach Ostdeutschland, insbesondere zu Offiziellen, eine kritische Angelegenheit. Bock versuchte dennoch, den Graben zwischen Ost und West nicht tiefer werden zu lassen. Seine unbestrittenen Kontakte zur DDR-Gewerkschaft und seine Verteidigung der Gespräche von Trost und Knepper mit Ostfunktionären (siehe Kapitel 3.2.3) sind auf Bocks ostdeutsche Herkunft zurückzuführen. Er wollte die Tür für ein vereinigtes Deutschland offenhalten. Umso enttäuschter zeigte er sich nach dem Bau der Mauer:

»Wir blicken auf die Berliner Schandmauer, die jede Aussicht auf eine Wiedervereinigung in absehbarer Zeit vollends zerschlagen hat. ${ }^{110}$

Während der ersten zehn Jahre der GTB gab es im GHV eine große personelle Kontinuität. In den fünf zweijährigen Wahlperioden bis 1959 gab es im GHV nur zwei personelle Veränderungen: 1951 wurde Karl Buschmann gewählt und 1957 ersetzte der Christdemokrat Karl Dörpinghaus den zum DGB gewechselten Bernhard Tacke. Nachdem sich Werner Bock als Gründungsvater 1949 in einer Kampfabstimmung mit zwei Drittel der Stimmen auch als Vorsitzender durchgesetzt hatte, wurde er sechsmal wiedergewählt. Die Wahlen auf den Gewerkschaftstagen 1951, 1953 und 1955 erfolgten per Akklamation, obwohl die Satzung eine geheime Wahl vorschrieb. Diesen mehrfachen Satzungsverstoß rechtfertigte man durch eine jeweils vorangehende Abstimmung über dieses Verfahren. Erst 1957 wurde der Vorsitzende wieder in geheimer Wahl gewählt. Bock erhielt 71,6 Prozent der Stimmen, 1959 waren es 83,5 Prozent und 1961 bei seiner letzten Wahl 82,2 Prozent der abgegebenen Delegiertenstimmen.

Seinen systemkritischen und gegenüber den Arbeitgebern grundsätzlich konfrontativen Kurs über sein Amtsende hinaus zu sichern gelang Werner Bock nicht. Die Kritiker des traditionellen Kurses hatten zwei objektive Schwachpunkte ausgemacht, zum einen die unbefriedigende Mitgliederentwicklung, zum anderen das autoritäre Auftreten des Vorsitzenden.

Dem erheblichen Mitgliederzuwachs bis 1952 folgte ein kontinuierlicher Rückgang. Die großen Arbeitskämpfe in den 1950er Jahren wirkten sich nur in den direkt vom Streik betroffenen Regionen positiv auf die Mitgliederentwicklung aus, d.h. die dortigen Mitgliederzuwächse der Streikjahre 1953, 1955 und 1958 strahlten nicht auf das gesamte Bundesgebiet aus. Der Mitgliederverlust insbesondere durch in Krisenzeiten entlassene Arbeiter:innen konnte in Zeiten von Neueinstellungen nicht durch neu geworbene Mitglieder wettgemacht werden. Trotz aller Schwankungen stieg die Zahl der Beschäftigten von 1952 bis 1960 um 155.000,

110 textil-bekleidung, Ausgabe 1/1962, S. 3. 
während die Mitgliederzahl im gleichen Zeitraum um 75.000 sank. Bock nannte drei Gründe für diesen Mitgliederrückgang im zweistelligen Prozentbereich: den Druck der Arbeitgeber auf Beschäftigte, der Gewerkschaft fernzubleiben, bessere betriebliche Sozialleistungen, aber auch eine offensichtlich mangelnde Mitgliederbetreuung. ${ }^{11}$

Zum anderen störten sich vor allem Jüngere am autoritären Auftreten von Werner Bock und dem nach heutigen Maßstäben mangelnden Demokratieverständnis, das er und seine Mitstreiter:innen an den Tag legten. Bock hielt eine starke Führung der Gewerkschaft für notwendig, denn nur durch vereintes Handeln könne sie ihre Durchsetzungsfähigkeit erhalten. Bis zum Ende seiner Amtszeit wurde er von der Mehrheit in Hauptvorstand und Beirat gestützt, auch wenn die oppositionellen Stimmen in diesen Spitzengremien in der zweiten Hälfte der 1950er Jahre lauter wurden. Dass seine Mehrheit in den Spitzengremien nicht mehr der Stimmung in der Gewerkschaft insgesamt entsprach, nahm er nicht wahr. Die Kritiker waren in seinen Augen eine Minderheit, von der er Geschlossenheit einforderte. Nach dem Gewerkschaftstag 1959, auf dem nicht nur wegen der Trost-Knepper-Affäre deutliche Kritik an der Führung hörbar wurde, warnte Bock, dass

»aufbauende Cewerkschaftsarbeit, die uns der sozialen Gerechtigkeit näher bringt, nur auf dem Fundament einer einigen und festgefügten Cemeinschaft möglich ist.«112

Die Modernisierungsanliegen der »Reformer« hielt Bock für blauäugig. Dabei unterschätzte er die Kraft und das strategische Vorgehen der Opposition sowie das schwindende Vertrauen in die durch die Weimarer Zeit geprägten Funktionär:innen und unterlag schließlich auf dem Gewerkschaftstag 1961 deutlich mit seinem Personalvorschlag. Bei der Entscheidung der Delegierten spielte neben den objektiven Defiziten auch das Verhalten von Bock in Sachen Trost und Knepper eine große Rolle. Der Vorsitzende hatte sich zu einseitig auf deren Seite gestellt und durchaus trickreich ihre Rehabilitation forciert, von der er sich auch nach Anklageerhebung durch die Bundesanwaltschaft nicht abbringen ließ.

Bis zum Schluss blieb Werner Bock sich treu. Noch in seiner letzten Rede warnte er davor, Stil und neue Methoden im Umgang mit den Arbeitgebern überzubewerten. Bei allem Reformeifer dürfe nicht vergessen werden, »daß wir im letzten eine Kampforganisation sind ${ }^{113}$ Doch unterschätzten er und die »Tra-

111 GTB: Ceschäftsbericht 1953-1954 des Hauptvorstandes, S. 6.

112 textil-bekleidung, Ausgabe 1/1960, S. 3.

113 GTB: Protokoll des 8. Ordentlichen Cewerkschaftstages, 16.-20. September 1963 in Hannover, S. $57 f$. 
ditionalisten « in Hauptvorstand und Beirat den Veränderungswillen vor allem jüngerer hauptamtlicher Gewerkschafter, die ein halbes Jahrzehnt lang auf den Richtungswechsel hingearbeitet hatten. So wurde 1963 mit Karl Buschmann die Galionsfigur der »Modernisierer« zu seinem Nachfolger gewählt. Werner Bock starb nur wenige Monate nach seinem Ausscheiden aus dem Amt am 1. August 1964 in Bielefeld.

Auf dem Gewerkschaftstag 1963 vollzog die GTB auch einen Generationswechsel. Aus der Gründungszeit blieb nur noch der Hauptkassierer Paul Trost im GHV. Mit Josef Hoffmann (37 Jahre), Hermann Schumacher (35) und Martin Lange (39) sank das Durchschnittsalter im GHV deutlich. Hoffmann bekam die Zuständigkeit für Personal, Schumacher übernahm das Tarifressort, für den »Traditionalisten« Martin Lange blieben die Mitgliederwerbung, Frauen- und Jugendarbeit. Auch wenn sich der neue Kurs durchgesetzt hatte, bildeten diejenigen, die den sozialpartnerschaftlichen Ansätzen kritisch gegenüberstanden, eine starke Minderheit. Die Spaltung zwischen den »Reformern« und den »Traditionalisten« sollte die Organisation noch zwei Jahrzehnte lang beschäftigen. 